\title{
Thermodynamical Bethe Ansatz and Condensed Matter
}

\author{
Minoru TAKAHASHI \\ Institute for Solid State Physics, University of Tokyo, \\ Roppongi, Minato-ku, Tokyo 106, Japan
}

\begin{abstract}
The basics of the thermodynamic Bethe ansatz equation are given. The simplest case is repulsive delta function bosons, the thermodynamic equation contains only one unknown function. We also treat the XXX model with spin $1 / 2$ and the XXZ model and the XYZ model. This method is very useful for the investigation of the low temperature thermodynamics of solvable systems.
\end{abstract}




\section{Introduction}

Thermodynamic Bethe ansatz equations were first introduced by Yang and Yang for the model of repulsive delta-function bosons. Later this method was extended to the other Bethe ansatz soluble models such as the XXX model, the XXZ model, the XYZ model, delta-function fermions, the Hubbard model, the t-J model and so on. In these theories the 0 of strings and holes play an essential role. The number of kinds of strings is generally infinite except in some special cases. We can construct a set of non-linear integral equations for these models. These are very useful for the investigation of the low-temperature thermodynamics of soluble models. Nowadays thermodynamic Bethe ansatz equations have been derived for almost all solvable one-dimensional quantum models.

In chapter 2, Yang and Yang's theory for bosons is introduced. Here the number of unknown functions is 1. In chapter 3 we treat the XXX model. Here the number of unknown functions is infinite. But we can treat this set of integral equations analytically in some special cases such as the infinite temperature limit, the zero temperature limit, and the high magnetic field limit. In chapter 3 we treat the XXZ model. The case $|\Delta|>1$ is almost identical to the XXX model $(\Delta=1)$. On the other hand the case $|\Delta|<1$ is more complicated, the number of kinds of strings is dependent on a parameter. If $\left(\cos ^{-1} \Delta\right) / \pi$ is a rational number, the number of kinds of strings is finite and thermodynamic Bethe ansatz equations contain a finite number of unknown functions. In chapter 5 we treat the XYZ model in zero magnetic field. Here, the number of unknown functions is also dependent on parameters. The equations are obtained by a slight modification of XXZ model at $|\Delta|<1$. In chapter 6 we review numerical calculations of these Bethe ansatz equations. 


\section{Repulsive delta-function bosons}

\section{Bethe ansatz equations and uniqueness of the solution}

Here we consider the system

$$
\mathcal{H}=-\sum_{i=1}^{N} \frac{\partial^{2}}{\partial x_{i}^{2}}+2 c \sum_{i<j} \delta\left(x_{i}-x_{j}\right),
$$

the problem of bosons interacting via a repulsive delta function potential with periodic boundary conditions. Lieb and Liniger showed that this model is solvable by Bethe ansatz and calculated the ground state energy and elementary excitations (Lieb and Liniger (1963), Lieb (1963)). The wave function is assumed to be a linear combination of $N$ ! plane waves. One must determine $N$ ! coefficients $A(P)$ with eigenvalue $E$ at $x_{1} \leq x_{2} \leq \ldots \leq x_{N}$

$$
\begin{aligned}
& f=\sum_{P} A(P) \exp \left[i\left(k_{P 1} x_{1}+k_{P 2} x_{2}+\ldots+k_{P N} x_{N}\right)\right] \\
& A(P)=C \epsilon(P) \prod_{j<k}\left(k_{P j}-k_{P k}+i c\right) \\
& E=\sum_{j=1}^{N} k_{j}^{2}
\end{aligned}
$$

Here $P$ 's are permutations and $\epsilon(P)$ is the parity of permutation $P$. The total momentum is given by

$$
K=\sum_{j=1}^{N} k_{j} .
$$

$k_{j}$ 's are called the quasi momenta. If we apply periodic boundary conditions, $k_{j}$ 's should be solutions of the following coupled equations

$$
e^{i k_{j} L}=-\prod_{l=1}^{N}\left(\frac{k_{j}-k_{l}+i c}{k_{j}-k_{l}-i c}\right) .
$$

The logarithm of these equations is

$$
k_{j} L=2 \pi I_{j}-\sum_{l} 2 \tan ^{-1}\left(\frac{k_{j}-k_{l}}{c}\right) .
$$


Here the $I_{j}$ 's form a set of distinct but otherwise arbitrary integers (half-odd integers) for odd(even) $N$. The quasi-momenta $k_{j}$ are determined by eq. (4) if a set of quantum numbers $I_{j}$ is given. The ground state energy is obtained from a Fredholm type integral equation (Lieb and Liniger (1963), Lieb (1963)). For this model Yang and Yang found a non-linear integral equation which gives the free energy at a given temperature (Yang and Yang (1969)). This result is important because it forms the basic theory for thermodynamics of other solvable models.

At first we should show that the solution of equation (4) is unique for a given set of $I_{j}$ 's. The equations (4) are equivalent to the following equations

$$
\begin{aligned}
& \frac{\partial B\left(k_{1}, k_{2}, \ldots, k_{N}\right)}{\partial k_{j}}=0 \\
& B\left(k_{1}, k_{2}, \ldots, k_{N}\right) \equiv \frac{L}{2} \sum k_{j}^{2}-2 \pi I_{j} k_{j}+\sum_{j<l} \theta_{1}\left(k_{j}-k_{l}\right), \\
& \theta_{1}(x)=x \tan ^{-1}(x / c)-\frac{c}{2} \ln \left(1+(x / c)^{2}\right) .
\end{aligned}
$$

The extremal point of the function $B$ with $N$ variables is determined by the solution of (4). It gives an eigenstate of the Hamiltonian. Let us consider the following $N \times N$ matrix

$$
B_{j l}=\frac{\partial^{2} B}{\partial k_{j} \partial k_{l}}=\delta_{j l}\left(L+\sum_{m} \frac{2 c}{c^{2}+\left(k_{j}-k_{m}\right)^{2}}\right)-\frac{2 c}{c^{2}+\left(k_{j}-k_{l}\right)^{2}} .
$$

This matrix is always positive definite because

$$
\sum_{l j} u_{l} B_{l j} u_{j}=L \sum u_{l}^{2}+\sum_{l<j} \frac{2 c}{c^{2}+\left(k_{j}-k_{l}\right)^{2}}\left(u_{j}-u_{l}\right)^{2} \geq 0,
$$

for an arbitrary real vector $\left\{u_{j}\right\}$. The function $B$ is a concave function in $N$ dimensional space. Thus a solution of equation (4) for a given set of $\left\{I_{j}\right\}$ is unique.

\section{Holes of quasi momenta and their distribution function}

In the limit of $c \rightarrow \infty$ this set of eigenstates is complete. Let us consider the following function

$$
h(k)=k+\frac{2}{L} \sum_{j} \tan ^{-1} \frac{k-k_{j}}{c} .
$$

The position of a hole is defined by

$$
h\left(k_{h}\right)=\frac{2 \pi}{L} \times \text { unoccupied (half }- \text { odd) integer. }
$$

We define the distribution function of holes $\rho^{h}(k)$ such that the number of holes between $k$ and $k+d k$ is $\rho^{h}(k) L d k$ and the density of particles $\rho(k)$ such that the 
number of particles between $k$ and $k+d k$ is $\rho(k) L d k$. In the thermodynamic limit we have

$$
2 \pi \int^{k} \rho(t)+\rho^{h}(t) d t=h(k)=k+2 \int \tan ^{-1} \frac{k-k^{\prime}}{c} \rho\left(k^{\prime}\right) d k^{\prime} .
$$

Differentiating this yields

$$
2 \pi\left(\rho(k)+\rho^{h}(k)\right)=1+2 \int_{-\infty}^{\infty} \frac{c \rho\left(k^{\prime}\right) d k^{\prime}}{c^{2}+\left(k-k^{\prime}\right)^{2}} .
$$

From equation (2) one obtains the energy and the particle number per unit length

$$
e=\int_{-\infty}^{\infty} k^{2} \rho(k) d k, \quad n=\int_{-\infty}^{\infty} \rho(k) d k
$$

The entropy of the distribution between $k$ and $k+d k$ is the logarithm of number of orderings of $L \rho(k) d k$ particles and $L \rho^{h}(k) d k$ holes

$$
\begin{aligned}
\ln \frac{\left[L\left(\rho(k)+\rho^{h}(k)\right) d k\right] !}{[L \rho(k)] !\left[L \rho^{h}(k)\right] !} & =L d k\left[\left(\rho(k)+\rho^{h}(k)\right) \ln \left(\rho(k)+\rho^{h}(k)\right)\right. \\
& \left.-\rho(k) \ln \rho(k)-\rho^{h}(k) \ln \rho^{h}(k)\right] .
\end{aligned}
$$

Here we use the Stirling formula $\ln (n !) \simeq n(\ln n-1)$. Then the entropy per unit length is

$$
s=\int_{-\infty}^{\infty}\left(\rho(k)+\rho^{h}(k)\right) \ln \left(\rho(k)+\rho^{h}(k)\right)-\rho(k) \ln \rho(k)-\rho^{h}(k) \ln \rho^{h}(k) d k .
$$

\section{Thermodynamic equilibrium}

The free energy per unit length $f=e-T s$ must be minimized under the condition that $n$ is constant. $f$ and $n$ are functionals of $\rho(k)$ and $\rho^{h}(k)$. Thus we should determine $\rho(k)$ and $\rho^{h}(k)$ to minimize $f-A n$. Next we look for parameter $A$ such that $n$ takes on its required value. In variational calculus $A$ is called a Lagrange multiplier. At the minimum point, the variation of $f-A n$ must be zero for any infinitesimal variation of functions

$$
\begin{aligned}
& 0=\delta \int_{-\infty}^{\infty}\left(k^{2}-A\right) \rho(k)-T\left\{\left(\rho(k)+\rho^{h}(k)\right) \ln \left(\rho(k)+\rho^{h}(k)\right)\right. \\
& \left.-\rho(k) \ln \rho(k)-\rho^{h}(k) \ln \rho^{h}(k)\right\} d k \\
& =\int\left\{k^{2}-A-T \ln \left(\frac{\rho(k)+\rho^{h}(k)}{\rho(k)}\right)\right\} \delta \rho(k)-T \ln \left(\frac{\rho(k)+\rho^{h}(k)}{\rho^{h}(k)}\right) \delta \rho^{h}(k) d k .
\end{aligned}
$$

One should note that $\delta \rho(k)$ and $\delta \rho^{h}(k)$ are not independent. From (10) we have

$$
\delta \rho^{h}(k)=-\delta \rho(k)+\frac{1}{\pi} \int_{-\infty}^{\infty} \frac{c \delta \rho\left(k^{\prime}\right) d k^{\prime}}{c^{2}+\left(k-k^{\prime}\right)^{2}} .
$$


Substituting this into (13), one obtains

$$
\begin{aligned}
& 0=\int_{-\infty}^{\infty} \delta \rho(k) \\
& \times\left\{k^{2}-A-T \ln \left(\frac{\rho^{h}(k)}{\rho(k)}\right)-\frac{T}{\pi} \int_{-\infty}^{\infty} d q \frac{c}{c^{2}+(k-q)^{2}} \ln \left(1+\frac{\rho(q)}{\rho^{h}(q)}\right) d q\right\}
\end{aligned}
$$

This equation must hold for any arbitrary infinitesimal change of the function $\rho(k)$. Thus

$$
T \ln \left(\frac{\rho^{h}(k)}{\rho(k)}\right)=k^{2}-A-\frac{T}{\pi} \int_{-\infty}^{\infty} d q \frac{c}{c^{2}+(k-q)^{2}} \ln \left(1+\frac{\rho(q)}{\rho^{h}(q)}\right) d q,
$$

must be satisfied at thermodynamic equilibrium. If we put $\epsilon(k)=T \ln \left(\rho^{h}(k) / \rho(k)\right)$, this becomes

$$
\epsilon(k)=k^{2}-A-\frac{T}{\pi} \int_{-\infty}^{\infty} d q \frac{c}{c^{2}+(k-q)^{2}} \ln \left(1+\exp \left(-\frac{\epsilon(q)}{T}\right)\right) d q .
$$

$\epsilon(k)$ can be determined by iteration. This function has the physical meaning as the excitation energy for an elementary excitation, as will be shown in next section. From equation (10) we have

$$
2 \pi \rho(k)\left(1+\exp \left(\frac{\epsilon(k)}{T}\right)\right)=1+2 c \int_{-\infty}^{\infty} \frac{\rho(q) d q}{c^{2}+(k-q)^{2}} .
$$

From this equation we can determine $\rho(k)$.

If equation (16) is differentiated with respect to the chemical potential A, one obtains a linear integral equation

$$
\frac{\partial \epsilon(k, A)}{\partial A}=-1+\int d q \frac{c}{c^{2}+(k-q)^{2}} \frac{1}{1+\exp \left(\frac{\epsilon(k)}{T}\right)} \frac{\partial \epsilon(q, A)}{\partial A} .
$$

Comparing this with (17) one finds that

$$
\frac{\partial \epsilon(k, A)}{\partial A}=-2 \pi \rho(k)\left(1+\exp \left(\frac{\epsilon(k)}{T}\right)\right)=-2 \pi\left(\rho(k)+\rho^{h}(k)\right) .
$$

The entropy density (12) can be written

$$
s=\int\left[\left(\rho+\rho^{h}\right) \ln (1+\exp (-\epsilon(k) / T))+\rho(k) \epsilon(k) / T\right] d k .
$$

The free energy density is

$$
f=\int\left(k^{2}-\epsilon(k)\right) \rho(k)-T\left(\rho(k)+\rho^{h}(k)\right) \ln \left(1+\exp \left(-\frac{\epsilon(k)}{T}\right)\right) d k .
$$


Substituting (16) into (20) one obtains

$$
\begin{aligned}
& f=\int\left[A+\frac{T}{\pi} \int \frac{d q c}{c^{2}+(k-q)^{2}} \ln \left(1+\exp \left(-\frac{\epsilon(q)}{T}\right)\right)\right] \rho(k) \\
& -T\left(\rho(k)+\rho^{h}(k)\right) \ln \left(1+\exp \left(-\frac{\epsilon(k)}{T}\right)\right) d k \\
& =\int A \rho(k) d k-T \int \ln \left(1+\exp \left(-\frac{\epsilon(k)}{T}\right)\right) \\
& \times\left[\rho(k)+\rho^{h}(k)-\frac{1}{\pi} \int \frac{c}{c^{2}+(k-q)^{2}} \rho(q) d q\right] d k .
\end{aligned}
$$

Substituting (10) we have a very simple expression for the free energy density

$$
f=A n-T \int \frac{d k}{2 \pi} \ln \left(1+\exp \left(-\frac{\epsilon(k)}{T}\right)\right) .
$$

The thermodynamic potential density $g=f-A n$ is

$$
g(T, A)=-T \int \ln \left(1+\exp \left(-\frac{\epsilon(k)}{T}\right)\right) \frac{d k}{2 \pi} .
$$

For a given temperature $T$ and chemical potential $A, \epsilon(k)$ is determined through the non-linear integral equation (16) and obtain thermodynamic potential $g$ from (23). All thermodynamic quantities are derived from $g(T, A)$ through thermodynamic relations

$$
n=-\frac{\partial g}{\partial A}, \quad s=-\frac{\partial g}{\partial T}, \quad e=g+A n+T s, \ldots
$$

The pressure $p$ is $-g$.

\section{Elementary excitations}

We consider the change of energy and momentum when one particle is moved from the thermodynamic equilibrium. Assume that the $l$-th particle is removed and one particle is added between the $m$-th and $m+1$-th particle

$$
\begin{aligned}
& \left\{I_{1}>I_{2}>I_{3}>\ldots>I_{N}\right\} \rightarrow \\
& \left\{I_{1}>I_{2}>\ldots>I_{m}>I_{l}^{\prime}>I_{m+1} \ldots I_{l-1}>I_{l+1}>\ldots>I_{N}\right\}
\end{aligned}
$$

The Bethe ansatz equations for the original state are

$$
L k_{j}=2 \pi I_{j}-\sum_{n=1}^{N} 2 \tan ^{-1} \frac{k_{j}-k_{n}}{c} j=1, \ldots, N .
$$


For the excited state they are

$$
\begin{aligned}
& L k_{j}^{\prime}=2 \pi I_{j}-2 \tan ^{-1} \frac{k_{j}^{\prime}-k_{l}^{\prime}}{c}-\sum_{n \neq m} 2 \tan ^{-1} \frac{k_{j}^{\prime}-k_{n}^{\prime}}{c}, j \neq l \\
& L k_{l}^{\prime}=2 \pi I_{l}^{\prime}-\sum_{n \neq m} 2 \tan ^{-1} \frac{k_{l}^{\prime}-k_{n}^{\prime}}{c} .
\end{aligned}
$$

The change of momentum and energy are

$$
\begin{aligned}
& \Delta K=k_{l}^{\prime}-k_{l}+\sum_{n \neq l}\left(k_{n}^{\prime}-k_{n}\right), \\
& \Delta E=k_{l}^{\prime 2}-k_{l}^{2}+\sum_{n \neq l}\left(k_{n}^{\prime 2}-k_{n}^{2}\right)
\end{aligned}
$$

Subtracting (25) from (26) we have

$$
\begin{aligned}
& \Delta k_{j} L+\sum \frac{2 c}{c^{2}+\left(k_{j}-k_{l}\right)^{2}}\left(\Delta k_{j}-\Delta k_{l}\right) \\
& =2 \tan ^{-1} \frac{k_{j}-k_{l}}{c}-2 \tan ^{-1} \frac{k_{j}-k_{l}^{\prime}}{c} .
\end{aligned}
$$

Then equation for the back-flow $J(k)=\rho(k) \Delta k L$ is

$$
\begin{aligned}
& \left(1+\frac{\rho^{h}(k)}{\rho(k)}\right) J\left(k ; k_{l}^{\prime}, k_{l}\right)-\int \frac{c}{\pi\left(c^{2}+\left(k-k^{\prime}\right)^{2}\right)} J\left(k^{\prime} ; k_{l}^{\prime}, k_{l}\right) d k^{\prime} \\
& =\frac{1}{\pi}\left(\tan ^{-1} \frac{k-k_{l}}{c}-\tan \frac{k-k_{l}^{\prime}}{c}\right) .
\end{aligned}
$$

Energy and momentum changes are

$$
\begin{aligned}
& \Delta K\left(k_{l}^{\prime}, k_{l}\right)=k_{l}^{\prime}-k_{l}+\int J\left(k ; k_{l}^{\prime}, k_{l}\right) d k, \\
& \Delta E\left(k_{l}^{\prime}, k_{l}\right)=k_{l}^{\prime 2}-k_{l}^{2}+\int 2 k J\left(k ; k_{l}^{\prime}, k_{l}\right) d k .
\end{aligned}
$$

It is clear that $\Delta K\left(k_{l}, k_{l}\right)=\Delta E\left(k_{l}, k_{l}\right)=0$. The differentiation of these with respect to $k_{l}^{\prime}$ is

$$
\begin{aligned}
& \frac{\partial \Delta K}{\partial k_{l}^{\prime}}=1+\int u\left(k ; k_{l}^{\prime}\right) d k \\
& \frac{\partial \Delta E}{\partial k_{l}^{\prime}}=2 k_{l}^{\prime}+\int 2 k u\left(k ; k_{l}^{\prime}\right) d k,
\end{aligned}
$$


where

$$
\begin{aligned}
& u\left(k ; k_{l}^{\prime}\right) \equiv \frac{\partial}{\partial k_{l}^{\prime}} J\left(k ; k_{l}^{\prime}, k_{l}\right), \\
& (1+\eta(k)) u\left(k ; k_{l}^{\prime}\right)-\int a\left(k-k^{\prime}\right) u\left(k^{\prime} ; k_{l}^{\prime}\right) d k^{\prime}=a\left(k-k_{l}^{\prime}\right) .
\end{aligned}
$$

Using this integral equation we find that $u\left(k ; k_{l}^{\prime}\right)$ is given by the infinite series

$$
\begin{aligned}
& u\left(k ; k_{l}^{\prime}\right)=\frac{1}{1+\eta(k)}\left[a\left(k-k_{l}^{\prime}\right)+\int d k_{1} \frac{a\left(k-k_{1}\right)}{1+\eta\left(k_{1}\right)} a\left(k_{1}-k_{l}^{\prime}\right)\right. \\
& \left.+\iint d k_{1} d k_{2} \frac{a\left(k-k_{1}\right)}{1+\eta\left(k_{1}\right)} \frac{a\left(k_{1}-k_{2}\right)}{1+\eta\left(k_{2}\right)} a\left(k_{2}-k_{l}^{\prime}\right)+\ldots\right] .
\end{aligned}
$$

Substituting this into (32) we get an expression of $\frac{\partial \Delta K}{\partial k_{l}^{\prime}}$ and $\frac{\partial \Delta E}{\partial k_{l}^{\prime}}$ as an infinite series

$$
\begin{aligned}
& \frac{\partial \Delta K}{\partial k_{l}^{\prime}}=1+\int d k_{1} a\left(k_{l}^{\prime}-k_{1}\right) \frac{1}{1+\eta\left(k_{1}\right)} \\
& +\iint d k_{1} d k_{2} a\left(k_{l}^{\prime}-k_{1}\right) \frac{1}{1+\eta\left(k_{1}\right)} a\left(k_{1}-k_{2}\right) \frac{1}{1+\eta\left(k_{2}\right)}+\ldots \\
& \frac{\partial \Delta E}{\partial k_{l}^{\prime}}=2 k_{l}^{\prime}+\int d k_{1} a\left(k_{l}^{\prime}-k_{1}\right) \frac{1}{1+\eta\left(k_{1}\right)} 2 k_{1} \\
& +\iint d k_{1} d k_{2} a\left(k_{l}^{\prime}-k_{1}\right) \frac{1}{1+\eta\left(k_{1}\right)} a\left(k_{1}-k_{2}\right) \frac{1}{1+\eta\left(k_{2}\right)} 2 k_{2}+\ldots
\end{aligned}
$$

From these infinite series linear integral equations for $\frac{\partial \Delta K}{\partial k_{l}^{\prime}}$ and $\frac{\partial \Delta E}{\partial k_{l}^{\prime}}$ are obtained,

$$
\begin{gathered}
\frac{\partial \Delta K\left(k_{l}^{\prime}, k_{l}\right)}{\partial k_{l}^{\prime}}=1+\int a\left(k_{l}^{\prime}-k_{1}\right) \frac{1}{1+\eta\left(k_{1}\right)} \frac{\partial \Delta K\left(k_{1}, k_{l}\right)}{\partial k_{1}}, \\
\frac{\partial \Delta E\left(k_{l}^{\prime}, k_{l}\right)}{\partial k_{l}^{\prime}}=2 k_{l}^{\prime}+\int a\left(k_{l}^{\prime}-k_{1}\right) \frac{1}{1+\eta\left(k_{1}\right)} \frac{\partial \Delta E\left(k_{1}, k_{l}\right)}{\partial k_{1}} .
\end{gathered}
$$

On the other hand we have equations for $2 \pi\left(\rho(k)+\rho^{h}(k)\right)$ and $\partial \epsilon(k) / \partial k$ from (10) and (16)

$$
\begin{aligned}
& 2 \pi\left(\rho(k)+\rho^{h}(k)\right)=1+\int a\left(k-k^{\prime}\right) \frac{2 \pi\left(\rho\left(k^{\prime}\right)+\rho^{h}\left(k^{\prime}\right)\right)}{1+\eta\left(k^{\prime}\right)} d k^{\prime}, \\
& \frac{\partial \epsilon(k)}{\partial k}=2 k+\int a\left(k-k^{\prime}\right) \frac{1}{1+\eta\left(k^{\prime}\right)} \frac{\partial \epsilon\left(k^{\prime}\right)}{\partial k^{\prime}} d k^{\prime} .
\end{aligned}
$$

The solution of non-singular linear integral equation is unique. Thus one obtains

$$
\begin{aligned}
& \frac{\partial \Delta K\left(k_{l}^{\prime}, k_{l}\right)}{\partial k_{l}^{\prime}}=2 \pi\left(\rho\left(k_{l}^{\prime}\right)+\rho^{h}\left(k_{l}^{\prime}\right)\right), \\
& \frac{\partial \Delta E\left(k_{l}^{\prime}, k_{l}\right)}{\partial k_{l}^{\prime}}=\frac{\partial \epsilon\left(k_{l}^{\prime}\right)}{\partial k_{l}^{\prime}} .
\end{aligned}
$$


Integrating these we have

$$
\begin{aligned}
& \Delta K\left(k_{l}^{\prime}, k_{l}\right)=2 \pi \int_{k_{l}}^{k_{l}^{\prime}} \rho(k)+\rho^{h}(k) d k, \\
& \Delta E\left(k_{l}^{\prime}, k_{l}\right)=\epsilon\left(k_{l}^{\prime}\right)-\epsilon\left(k_{l}\right) .
\end{aligned}
$$

Thus $\epsilon(k)=T \ln \left(\rho^{h}(k) / \rho(k)\right)$ has the physical meaning as the energy of the elementary excitation.

\section{Some special limits}

\section{$5.1 c=\infty$ limit}

In the thermodynamic limit the system is equivalent to ideal spinless fermions

$$
\begin{aligned}
& p=\frac{1}{2 \pi} \int \frac{k^{2} d k}{\exp \left(k^{2}-A\right) / T+1}= \\
& -\frac{T}{2 \pi} \int \ln \left(1+\exp \left[\left(A-k^{2}\right) / T\right]\right) d k=\lim _{L \rightarrow \infty}-G / L,
\end{aligned}
$$

by the partial differentiation with respect to $k$. In this limit $c /\left(c^{2}+(k-q)^{2}\right)$ in the integrand of (16) is zero. Then we have have very simple solution $\epsilon(k)=k^{2}-A$. From equation (17) we have

$$
\rho(k)=\frac{1}{2 \pi} \frac{1}{\exp \left[\left(k^{2}-A\right) / T\right]+1} .
$$

From (23) the thermodynamic potential per unit length is

$$
g=-\frac{1}{2 \pi} \int d k \ln \left(1+\exp \left(-\frac{k^{2}-A}{T}\right)\right) .
$$

This is equivalent to (39).

\section{$5.2 c=0+$ limit}

In this limit integration kernel $c /\left(c^{2}+(k-q)^{2}\right)$ can be replaced by $\pi \delta(k-q)$. Then (16) becomes $\epsilon(k)=k^{2}-A-T \ln (1+\exp (-\epsilon(k) / T))$. Then we obtain

$$
\epsilon(k)=T \ln \left(\exp \left(\left(k^{2}-A\right) / T\right)-1\right) .
$$

The Gibbs free energy per site $g$, pressure $p, \rho(k)$ and $\rho^{h}(k)$ are

$$
\begin{gathered}
g=-p=T \int \ln \left(1-\exp \left(-\frac{k^{2}-A}{T}\right)\right) \frac{d k}{2 \pi}, \quad \rho^{h}(k)=\frac{1}{2 \pi}, \\
\rho(k)=\frac{1}{2 \pi} \frac{1}{\exp \left(\left(k^{2}-A\right) / T\right)-1} .
\end{gathered}
$$

This result coincide with that for ideal bosons. 


\section{3 $T=0+$ limit}

Generally speaking $\epsilon(k)$ is a monotonically increasing function of $k^{2}$. At $T=0+$ we assume that $\epsilon\left( \pm q_{0}\right)=0$. Thus we have

$$
\rho(k)=0 \quad \text { for } k^{2}>q_{0}^{2}, \quad \rho^{h}(k)=0 \text { for } \quad k^{2}<q_{0}^{2} .
$$

Equations (10) and (16) for $k<q_{0}$ are

$$
\begin{aligned}
& 2 \pi \rho(k)=1+2 c \int_{-q_{0}}^{q_{0}} \frac{\rho(q) d q}{c^{2}+(k-q)^{2}}, \\
& \epsilon(k)=k^{2}-A+\frac{c}{\pi} \int_{-q_{0}}^{q_{0}} \frac{\epsilon(q) d q}{c^{2}+(k-q)^{2}} .
\end{aligned}
$$

The first equation is equivalent with Lieb-Liniger equation(Lieb and Liniger (1963)),

$$
\rho(k)=\frac{1}{2 \pi}+\int_{-B}^{B} \frac{c / \pi}{c^{2}+(k-q)^{2}} \rho(q) d q .
$$

The above theory was introduced by Yang-Yang (Yang and Yang (1969)). C.P. Yang solved numerically this equation(C.P. Yang 1970). Very surprisingly it seems that this simple non-linear equation gives the exact free energy in the thermodynamic limit of the 1D repulsive Bosons. The next problem is to find thermodynamic Bethe ansatz equation for other soluble models. As the next simplest case we treat the $S=1 / 2 \mathrm{XXX}$ chain. 


\section{Thermodynamics of the XXX chain}

\section{Wave functions of the XXX chain}

The Heisenberg model was the first model to be treated by the method of Bethe ansatz (Bethe (1931)). In the beginning of the 1930's only the ferromagnetic case was considered

$$
\begin{aligned}
& \mathcal{H}=-J \sum_{l=1}^{N} S_{l}^{x} S_{l+1}^{x}+S_{l}^{y} S_{l+1}^{y}+S_{l}^{z} S_{l+1}^{z}-2 h \sum_{l=1} S_{l}^{z}, \\
& h \geq 0, \quad \mathbf{S}_{N+1} \equiv \mathbf{S}_{1} .
\end{aligned}
$$

This Hamiltonian is defined on a $2^{N}$ dimensional vector space. The space is classified by the total $S^{z}=\sum S_{l}^{z}$. The ground state is the state where all spins are up and $S^{z}=N / 2$

$$
\mathcal{H}\left|0>=E_{0}\right| 0>, \quad E_{0}=-J N / 4-N h .
$$

Write a general state $\mid \Psi>$ in terms of a wave function $f$,

$$
\left|\Psi>=\sum f\left(n_{1}, n_{2}, \ldots, n_{M}\right) S_{n_{1}}^{-} S_{n_{2}}^{-} \ldots S_{n_{M}}^{-}\right| 0>,
$$

where $1 \leq n_{1}<n_{2}<\ldots<n_{M} \leq N$ and $2 M \leq N$. The eigenvalue equation is

$$
\begin{aligned}
& -\frac{J}{2} \sum_{j}\left(1-\delta_{n_{j}+1, n_{j+1}}\right)\left\{f\left(n_{1}, \ldots, n_{j}+1, n_{j+1}, \ldots, n_{M}\right)\right. \\
& \left.+f\left(n_{1}, \ldots, n_{j}, n_{j+1}-1, \ldots, n_{m}\right)\right\} \\
& +\left\{E_{0}-E+(J+2 h) M-J \sum_{j} \delta_{n_{j}+1, n_{j+1}}\right\} f\left(n_{1}, n_{2}, \ldots, n_{M}\right)=0 .
\end{aligned}
$$

Next we assume that the wave function is of the following form

$$
f\left(n_{1}, n_{2}, \ldots, n_{M}\right)=\sum_{P}^{M !} A(P) \exp \left(i \sum_{j=1}^{M} k_{P j} n_{j}\right) .
$$


Choosing

$$
\begin{aligned}
& A(P)=\epsilon(P) \prod_{j<l}\left(e^{i\left(k_{P j}+k_{P l}\right.}+1-2 e^{i k_{P l}}\right), \\
& E-E_{0}=2 h M+J \sum_{j=1}^{M}\left(1-\cos k_{j}\right) .
\end{aligned}
$$

insures that $f$ satisfies (49). If we put $e^{i k_{j}}=\left(x_{j}+i\right) /\left(x_{j}-i\right)$, the wave function and energy are written as follows

$$
\begin{aligned}
& f\left(n_{1}, n_{2}, \ldots, n_{M}\right)=\sum_{P}^{M !} A(P) \prod_{j=1}^{M}\left(\frac{x_{P j}+i}{x_{P j}-i}\right)^{n_{j}}, \\
& A(P)=D \epsilon(P) \prod_{j<l}\left(x_{P j}-x_{P l}-2 i\right), \\
& E-E_{0}=2 h M+2 J \sum_{j=1}^{M} \frac{1}{x_{j}^{2}+1} .
\end{aligned}
$$

The $x_{j}$ 's are called rapidities. The periodic boundary condition

$$
f\left(x_{1}, x_{2}, \ldots, x_{M}\right)=f\left(x_{2}, x_{3}, \ldots, x_{M}, x_{1}+N\right),
$$

implies the $x_{j}$ satisfy

$$
\left(\frac{x_{j}+i}{x_{j}-i}\right)^{N}=\prod_{l \neq j}\left(\frac{x_{j}-x_{l}+2 i}{x_{j}-x_{l}-2 i}\right), \quad j=1, \ldots, M
$$

In terms of the rapidity variable, the periodic boundary conditions take on a very simple form.

\section{Hulthen's solution for the antiferromagnet}

In actual magnetic substances, the ferromagnetic case is rare. Usual one- dimensional magnetic substances are antiferromagnetic (the $J<0$ case of equation (46)). The logarithm of equation (56) is

$$
2 N \tan ^{-1} x_{j}=2 \pi I_{j}+2 \sum_{l=1}^{M} \tan ^{-1} \frac{x_{j}-x_{l}}{2},
$$

where $I_{j}$ is an integer(half-odd integer) for odd(even) $N-M$. The total momentum is given by

$$
K=\pi\left(1-(-1)^{M}\right) / 2-\frac{2 \pi}{N} \sum_{j} I_{j} .
$$


For simplicity we set $N$ to be even. One can show that the lowest energy state in the subspace of total $S_{z}=N / 2-M$ is given by

$$
I_{j}=(M+1-2 j) / 2, \quad j=1,2, \ldots, M .
$$

In the thermodynamic limit $x_{j}$ 's distribute from $-B$ to $B$. From equation (57) we have

$$
\tan ^{-1} x=\pi \int^{x} \rho(t) d t+\int_{-B}^{B} \tan ^{-1} \frac{x-y}{2} \rho(y) d y .
$$

Differentiating with respect to $x$ yields

$$
\rho(x)=\frac{1}{\pi} \frac{1}{x^{2}+1}-\int_{-B}^{B} \frac{1}{\pi} \frac{2}{(x-y)^{2}+4} \rho(y) d y .
$$

The energy and magnetization per site are

$$
\begin{gathered}
\frac{E}{N}=\frac{|J|}{4}-h+\int_{-B}^{B}\left[2 h-\frac{2|J|}{x^{2}+1}\right] \rho(x) d x, \\
\frac{S_{z}}{N}=\frac{1}{2}-\int_{-B}^{B} \rho(x) d x .
\end{gathered}
$$

This integral equation can be solved in the case of infinite $B$. We define the Fourier transform of $\rho(x)$ as follows

$$
\tilde{\rho}(\omega)=\int_{-\infty}^{\infty} e^{-i \omega x} \rho(x) d x .
$$

Using the formula $\int \pi^{-1} n /\left(x^{2}+n^{2}\right) \exp (-i x \omega) d x=\exp (-n|\omega|)$, one can rewrite (60) as follows

$$
\rho(\omega)\left(1+e^{-2|\omega|}\right)=e^{-|\omega|} .
$$

Thus $\tilde{\rho}(\omega)=1 /(2 \cosh \omega)$ and

$$
\rho(x)=\frac{1}{2 \pi} \int_{-\infty}^{\infty} e^{i \omega x} \tilde{\rho}(\omega) d \omega=\frac{1}{4} \operatorname{sech}\left(\frac{\pi x}{2}\right) .
$$

Substituting this into (62) we have

$$
e=-|J|\left(\ln 2-\frac{1}{4}\right)=-0.443147|J|, \quad s_{z}=0 .
$$

It can be shown that the case $B=\infty$ is the true ground state at $h=0$ and that the magnetization is zero. Thus an analytical result for a one-dimensional antiferromagnet is obtained (Hulthen (1938)). The first neighbor correlation is derived from this result

$$
<S_{i}^{z} S_{i+1}^{z}>=\frac{1}{12}(1-4 \ln 2)=-0.14771573 .
$$


An analytic expression for the second neighbor correlation function is also known for this model (Takahashi (1977)),

$$
<S_{i}^{z} S_{i+2}^{z}>=\frac{1}{12}(1-16 \ln 2+9 \zeta(3))=0.06067977 .
$$

This was calculated from the ground state energy of the Hubbard model.

Hulthen's solution is the ground state of XXX antiferromagnet at zero magnetic field. Griffiths calculated the magnetization curve of this model (Griffiths (1964)). des Cloizeaux and Pearson calculated the elementary excitation away from this ground state (des Cloizeaux and Pearson (1962)).

\section{String solution of infinite system}

The elementary excitation for the $M=1$ case is

$$
E-E_{0}=J(1-\cos K)+2 h .
$$

This is the spin wave excitation of the ferromagnetic Heisenberg model. Bethe found that the bound state of spin waves exists (Bethe, 1931),

$$
x_{j}=\alpha+i(n+1-2 j), j=1,2, \ldots, n .
$$

The energy and momentum of this excitation are

$$
E=E_{0}+\frac{2 n J}{\alpha^{2}+n^{2}}+2 n h, \quad K=\frac{1}{i} \ln \left(\frac{\alpha+i n}{\alpha-i n}\right) .
$$

Thus the dispersion relation is

$$
E=E_{0}+\frac{2 J}{n}(1-\cos K)+2 n h .
$$

These string solutions play an essential role in the thermodynamics of soluble models. The author obtained the thermodynamic Bethe ansatz equation for the XXX model (Takahashi (1971a)). The wave function is

$$
\begin{aligned}
& f\left(n_{1}, n_{2}, \ldots, n_{M}\right)=\sum_{P} A(P) \prod_{j=1}^{M}\left(\frac{x_{P j}+i}{x_{P j}-i}\right)^{n_{j}}, \\
& A(P)=D \epsilon(P) \prod_{j<l}\left(x_{P j}-x_{P l}-2 i\right) .
\end{aligned}
$$

We consider the wave function in the equation (72) assuming that $N$ is infinity. Particle coordinates $n_{j}$ move from $-\infty$ to $\infty$. Assume that $\Im k_{1} \geq \Im k_{2} \geq \ldots \geq$ $\Im k_{n}$. The wave function is written as follows

$$
\begin{aligned}
& f\left(n_{1}, n_{2}, \ldots, n_{M}\right)=\left(z_{1} z_{2} . . z_{M}\right)^{n_{1}} \sum_{P} A(P) \prod_{j=2}^{M}\left(\prod_{l=j}^{M} z_{P l}\right)^{n_{j+1}-n_{j}}, \\
& A(P)=\epsilon(P) \prod_{j<l}\left(x_{P j}-x_{P l}-2 i\right), \quad z_{j}=e^{i k_{j}}=\left(\frac{x_{j}+i}{x_{j}-i}\right) .
\end{aligned}
$$


In the infinite system $f$ satisfies the following boundary conditions

$$
\begin{aligned}
& \left|\lim _{n_{1}, \ldots, n_{r} \rightarrow-\infty} f\left(n_{1}, n_{2}, \ldots, n_{M}\right)\right|<\infty, \\
& \left|\lim _{n_{M-r+1}, \ldots, n_{M} \rightarrow \infty} f\left(n_{1}, n_{2}, \ldots, n_{M}\right)\right|<\infty,
\end{aligned}
$$

From this condition we find that $\left|z_{1} z_{2} . . z_{M}\right|=1$ and that $A(P)=0$ if one of $\left|\prod_{l=j}^{M} z_{P l}\right|$ is greater than 1 . From the normalizability condition of the wave function we have

$$
A(I) \neq 0, \quad A(P \neq I)=0, \quad\left|z_{1} z_{2} \ldots z_{n}\right|=1 .
$$

These conditions are satisfied only if

$$
x_{j}=\alpha+(n+1-2 j) i, \quad j=1,2, \ldots, n .
$$

From $\left|z_{1} z_{2} . . z_{M}\right|=\left|\frac{\alpha+n i}{\alpha-n i}\right|=1$, we find that $\alpha$ must be real. The condition

$$
\left(\prod_{l=j+1}^{M} z_{l}\right)=\left|\frac{\alpha+(n-2 j) i}{\alpha-n i}\right| \leq 1, \quad j=1,2, \ldots, n-1
$$

is automatically satisfied for general $n$. Thus strings with arbitrary length are possible for the XXX Heisenberg chain. Moreover, the following type of string is impossible

$$
x_{1}=\alpha+(2+\beta) i, x_{2}=\alpha+\beta i, x_{3}=\alpha-\beta i, x_{4}=\alpha-(2+\beta) i,
$$

where $\alpha, \beta$ are real and $\beta \neq 1$.

\section{$9 \quad$ String hypothesis for a long XXX ring}

We assume that all rapidities $x_{j}$ belong to bound states with $n=1,2, \ldots$. For a bound states of $n$-x's the real parts of all $x$ 's are the same and imaginary parts are $(n-1) i,(n-3) i, \ldots,-(n-1) i$ within the accuracy of $O(\exp (-\delta N))$. This assumption seems to be too strong and there are some counter examples in special cases. This is a very controversial point of thermodynamic Bethe-ansatz equations for soluble models, except in the repulsive Boson case, which has no the string solution. But equations obtained using the string hypothesis seem to give the correct free energy and other thermodynamic quantities.

Consider the case where $M_{n}$ bound states of $n-x$ 's exist. We designate $x$ 's as

$$
\begin{gathered}
x_{\alpha}^{n, j}, \quad \alpha=1,2, \ldots, M_{n} \\
x_{\alpha}^{n, j}=x_{\alpha}^{n}+i(n+1-2 j)+\text { deviation. }
\end{gathered}
$$

From (56),

$$
e^{N}\left(x_{\alpha}^{n, j}\right)=\prod_{(m, \beta) \neq(n, \alpha)} e\left(\frac{x_{\alpha}^{n, j}-x_{\beta}^{m}}{m-1}\right) e\left(\frac{x_{\alpha}^{n, j}-x_{\beta}^{m}}{m+1}\right) \prod_{j^{\prime} \neq j} e\left(\frac{x_{\alpha}^{n, j}-x_{\alpha}^{n, j^{\prime}}}{2}\right),
$$




$$
j=1,2, \ldots, n .
$$

Here $e(x) \equiv(x+i) /(x-i)$. The last product is delicate because the numerator or denominator may become very small. If we take the product of these $n$ equations, these delicate terms are canceled and we have

$$
e^{N}\left(x_{\alpha}^{n} / n\right)=\prod_{j=1}^{n} e^{N}\left(x_{\alpha}^{n, j}\right)=\prod_{(m, \beta) \neq(n, \alpha)} E_{n m}\left(x_{\alpha}^{n}-x_{\beta}^{m}\right),
$$

where

$$
E_{n m}(x) \equiv\left\{\begin{array}{c}
e\left(\frac{x}{|n-m|}\right) e^{2}\left(\frac{x}{|n-m|+2}\right) e^{2}\left(\frac{x}{|n-m|+4}\right) \ldots e^{2}\left(\frac{x}{n+m-2}\right) e\left(\frac{x}{n+m}\right) \\
\text { for } n \neq m, \\
e^{2}\left(\frac{x}{2}\right) e^{2}\left(\frac{x}{4}\right) \ldots e^{2}\left(\frac{x}{2 n-2}\right) e\left(\frac{x}{2 n}\right) \text { for } n=m .
\end{array}\right.
$$

The logarithm of these equations gives

$$
N \theta\left(x_{\alpha}^{n} / n\right)=2 \pi I_{\alpha}^{n}+\sum_{(m, \beta) \neq(n, \alpha)} \Theta_{n m}\left(x_{\alpha}^{n}-x_{\beta}^{m}\right),
$$

where

$$
\theta(x) \equiv 2 \tan ^{-1}(x)
$$

and

$$
\Theta_{n m}(x) \equiv\left\{\begin{array}{l}
\theta\left(\frac{x}{n-m}\right)+2 \theta\left(\frac{x}{|n-m|+2}\right)+\ldots+2 \theta\left(\frac{x}{n+m-2}\right)+\theta\left(\frac{x}{n+m}\right) \\
\text { for } n \neq m, \\
2 \theta\left(\frac{x}{2}\right)+2 \theta\left(\frac{x}{4}\right)+\ldots+2 \theta\left(\frac{x}{2 n-2}\right)+\theta\left(\frac{x}{2 n}\right) \text { for } n=m .
\end{array}\right.
$$

$I_{\alpha}^{n}$ is an integer (half-odd integer) if $N-M_{n}$ is odd (even) and should satisfy

$$
\left|I_{\alpha}^{n}\right| \leq \frac{1}{2}\left(N-1-\sum_{m=1}^{\infty} t_{n m} M_{m}\right), \quad t_{n m} \equiv 2 \operatorname{Min}(n, m)-\delta_{n m} .
$$

We can prove the number of sets $\left\{I_{\alpha}^{n}\right\}$ is $C_{M}^{N}-C_{M-1}^{N}$, under the condition $M=$ $\sum_{n=1}^{\infty} n M_{n}$. Here $C_{M}^{N}$ is the binomial coefficient defined by $N ! /(M !(N-M) !)$. For details, see Appendix A of (Takahashi (1971a)). The energy of this state is given by

$$
E\left(\left\{I_{\alpha}^{n}\right\}\right)=N\left(-h-\frac{J}{4}\right)+\sum_{n, \alpha}\left(\frac{2 J n}{\left(x_{\alpha}^{n}\right)^{2}+n^{2}}+2 h n\right) .
$$

We can construct wave functions through (48), (50) and (54) at $S=S_{z}=N / 2-$ $M$. The wave functions for $S_{z}=S-1, S-2, \ldots,-S$ are obtained by applying the operator $S_{\text {total }}^{-}$. The energy for these cases are $E-2 h, E-4 h, \ldots, E-2(N-2 M) h$. Then the total number of states which are generated by the string assumption and the descending operator at $S_{z}=N / 2-M$ is $C_{M}^{N}$. Therefore the total number of states is $2^{N}$. This coincides with the true total number of states. It is expected 
that all eigen functions constructed in the above way should be a complete set. The partition function of this system is written as follows

$$
\mathcal{Z}=\sum_{M=0}^{[N / 2]} \frac{1-\exp (-2(N+1-2 M) h / T)}{1-\exp (-2 h / T)} \sum_{\left\{I_{\alpha}^{n}\right\}} \exp \left[-T^{-1} E\left(\left\{I_{\alpha}^{n}\right\}\right)\right] .
$$

The free energy is given by $G=-T \ln \mathcal{Z}$. We define functions $h_{n}(x)$ by

$$
h_{n}(x) \equiv \theta_{n}(x)-N^{-1} \sum_{(m, \alpha)} \Theta_{n m}\left(x-x_{\alpha}^{m}\right) .
$$

We can define holes in $n$-string sea by the solution of

$$
2 \pi J_{\beta}^{n} / N=h_{n}\left(x_{\beta}^{n}\right),
$$

where $J_{\beta}^{n}$ are omitted integers or half-odd integers in the region.

\section{Thermodynamic Bethe-ansatz equations for XXX chain}

In the thermodynamic limit, define distribution functions of $n$-strings and holes of $n$-string as $\rho_{n}(x)$ and $\rho_{n}^{h}(x)$. The number of strings and holes between $x$ and $x+d x$ is $\rho_{n}(x) N d x$ and $\rho_{n}^{h}(x) N d x$, respectively. Thus we have

$$
2 \pi \int^{x} \rho_{n}(t)+\rho_{n}^{h}(t) d t=\theta_{n}(x)-\sum_{m=1}^{\infty} \int_{-\infty}^{\infty} \Theta_{n m}(x-y) \rho_{m}(y) d y .
$$

Differentiating by $x$, we obtain the integral equation

$$
a_{n}(x)=\rho_{n}(x)+\rho_{n}^{h}(x)+\sum_{m} \int_{-\infty}^{\infty} T_{n m}(x-y) \rho_{m}(y) d y,
$$

where $T_{n m}(x)$ is a function defined by

$$
T_{n m}(x) \equiv\left\{\begin{array}{c}
a_{|n-m|}(x)+2 a_{|n-m|+2}(x)+2 a_{|n-m|+4}(x)+\ldots \\
+2 a_{n+m-2}(x)+a_{n+m}(x) \text { for } n \neq m, \\
2 a_{2}(x)+2 a_{4}(x)+\ldots+2 a_{2 n-2}(x)+a_{2 n}(x) \text { for } n=m .
\end{array}\right.
$$

$a_{n}(x)$ is a function defined by

$$
a_{n}(x) \equiv \frac{1}{\pi} \frac{n}{x^{2}+n^{2}}, \quad a_{0}(x) \equiv \delta(x) .
$$

The energy per site is

$$
\begin{aligned}
& e=-\left(\frac{J}{4}+h\right)+\sum_{n=1}^{\infty} \int_{-\infty}^{\infty} g_{n}(x) \rho_{n}(x) d x \\
& g_{n}(x) \equiv 2 \pi J a_{n}(x)+2 n h .
\end{aligned}
$$


The total entropy per site is

$$
s=\sum_{n=1}^{\infty} \int_{-\infty}^{\infty} \rho_{n}(x) \ln \left(1+\frac{\rho_{n}^{h}(x)}{\rho_{n}(x)}\right)+\rho_{n}^{h}(x) \ln \left(1+\frac{\rho_{n}(x)}{\rho_{n}^{h}(x)}\right) d x .
$$

$e-T s$ should be minimized at the thermodynamic equilibrium. Consider the functional variation of the free energy with respect to $\rho_{n}(k)$ and $\rho_{n}^{h}(k)$,

$$
\begin{aligned}
& 0=\delta e-T \delta s=\sum_{n=1}^{\infty} \int d x \\
& {\left[g_{n}(x)-T \ln \left(1+\frac{\rho_{n}^{h}(x)}{\rho_{n}(x)}\right)\right] \delta \rho_{n}(x)-T \ln \left(1+\frac{\rho_{n}(x)}{\rho_{n}^{h}(x)}\right) \delta \rho_{n}^{h}(x) .}
\end{aligned}
$$

From equation (88) we have

$$
\delta \rho_{n}^{h}(x)=-\delta \rho_{n}(x)-\sum_{m} \int T_{n m}(x-y) \delta \rho_{m}(y) d y .
$$

Substituting these into (96) yields

$0=T \sum_{n=1}^{\infty} \int\left\{\frac{g_{n}(x)}{T}-\ln \eta_{n}(x)+\sum_{m=1} \int T_{n m}(x-y) \ln \left(1+\eta_{m}^{-1}(y)\right) d y\right\} \delta \rho_{n}(x) d x$,

where $\eta_{n}(x) \equiv \rho_{n}^{h}(x) / \rho_{n}(x)$. Thus we have integral equations for an infinite number of unknown $\eta_{n}(x)$

$$
\ln \eta_{n}(x)=\frac{g_{n}(x)}{T}+\sum_{m=1}^{\infty} T_{n m} * \ln \left(1+\eta_{m}^{-1}(x)\right) .
$$

In the theory of Bethe ansatz equations we encounter very frequently the integration of the following type

$$
\int_{-\infty}^{\infty} a(x-y) b(y) d y
$$

This is a convolution of two functions $a(x)$ and $b(x)$. In the above and hereafter we write the convolution as $a * b(x)$,

$$
a * b(x) \equiv \int_{-\infty}^{\infty} a(x-y) b(y) d y .
$$

The free energy per site becomes as follows

$$
f=e-T s=-\left(\frac{J}{4}+h\right)+\sum_{n=1}^{\infty} \int g_{n} \rho_{n}-T\left[\rho_{n} \ln \left(1+\eta_{n}\right)+\rho_{n}^{h} \ln \left(1+\eta_{n}^{-1}\right)\right] d x .
$$


We eliminate $\rho_{n}^{h}$ using (91)

$$
\begin{aligned}
& f=-\left(\frac{J}{4}+h\right)-T \sum_{n=1}^{\infty} \int \ln \left(1+\eta_{n}^{-1}\right) a_{n}(x) \\
& +\rho_{n}\left[\ln \eta_{n}-\frac{g_{n}}{T}-T_{n m} * \ln \left(1+\eta_{m}^{-1}\right)\right] d x .
\end{aligned}
$$

Using (99), one sees the inside of the bracket on r.h.s. is zero. So we have

$$
f=-\left(\frac{J}{4}+h\right)-T \sum_{n=1}^{\infty} \int a_{n}(x) \ln \left(1+\eta_{n}^{-1}(x)\right) d x .
$$

From the $n=1$ case of (99) we have

$$
\ln \left(1+\eta_{1}\right)=\frac{2 \pi J a_{1}(x)+2 h}{T}+\sum_{l=1}^{\infty}\left(a_{l-1}+a_{l+1}\right) * \ln \left(1+\eta_{l}^{-1}\right) .
$$

Operating $\int d x s(x)$ on this equation yields

$$
\begin{gathered}
\int d x s(x) \ln \left(1+\eta_{1}\right)=\frac{2 \pi J}{T} \int s(x) a_{1}(x) d x+\frac{h}{T} \\
+\sum_{l=1}^{\infty} \int a_{l}(x) \ln \left(1+\eta_{l}^{-1}(x)\right) d x .
\end{gathered}
$$

Then equation (102) is transformed as follows

$$
f=J\left(\ln 2-\frac{1}{4}\right)-T \int s(x) \ln \left(1+\eta_{1}(x)\right) d x .
$$

Solutions $\eta_{n}$ of (99) are functions of $x, J, T$ and $h$. Differentiating (99) with respect to $J$ yields

$$
\frac{2 \pi J a_{n}(x)}{T}=\frac{1}{\eta_{n}} \frac{\partial \eta_{n}}{\partial J}+\sum_{m} T_{n m} * \frac{1}{\left(1+\eta_{m}\right) \eta_{m}} \frac{\partial \eta_{m}}{\partial J} .
$$

Comparing this with (91) we have

$$
\rho_{n}=\frac{T}{2 \pi} \frac{1}{\left(1+\eta_{n}\right) \eta_{n}} \frac{\partial \eta_{n}}{\partial J}, \quad \rho_{n}+\rho_{n}^{h}=\frac{T}{2 \pi} \frac{\partial \ln \eta_{n}}{\partial J} .
$$

By the definition (92),

$$
a_{1} *\left(T_{n-1, m}+T_{n+1, m}\right)-\left(a_{0}+a_{2}\right) * T_{n, m}=\left(\delta_{n-1, m}+\delta_{n+1, m}\right) a_{1} .
$$

Using equations (99), (107) yields

$$
\left(a_{0}+a_{2}\right) * \ln \eta_{1}(x)=\frac{2 \pi J a_{1}(x)}{T}+a_{1} * \ln \left(1+\eta_{2}(x)\right),
$$




$$
\left(a_{0}+a_{2}\right) * \ln \eta_{n}(x)=a_{1} * \ln \left(1+\eta_{n-1}(x)\right)\left(1+\eta_{n+1}(x)\right), \quad n=2,3, \ldots .
$$

Equations (108) and(109) are not complete to determine all of the $\eta_{n}(x)$, as they do not contain $h$. Take the $n=1$ case of (99)

$$
\ln \eta_{1}=\frac{2 \pi J a_{1}(x)+2 h}{T}+a_{2} * \ln \left(1+\eta_{1}^{-1}\right)+\sum_{j=2}^{\infty}\left(a_{j-1}+a_{j+1}\right) * \ln \left(1+\eta_{j}^{-1}\right)
$$

Substituting (108), (109) we can eliminate $\eta_{j}, j<n$ for a given integer $n$,

$$
\begin{aligned}
& \frac{2 h}{T}=a_{n} * \ln \eta_{n+1}-a_{n+1} * \ln \left(1+\eta_{n}\right)-a_{n+2} * \ln \left(1+\eta_{n+1}^{-1}\right) \\
& -\sum_{l=n+2}^{\infty}\left(a_{l-1}+a_{l+1}\right) * \ln \left(1+\eta_{l}^{-1}\right) .
\end{aligned}
$$

Thus we have

$$
\begin{aligned}
& \ln \eta_{n+1}=\frac{2 h}{T}+a_{1} * \ln \eta_{n}+a_{2} * \ln \left(1+\eta_{n+1}^{-1}\right) \\
& +\sum_{l=n+2}^{\infty}\left(a_{l-n-1}+a_{l-n+1}\right) * \ln \left(1+\eta_{l}^{-1}\right) .
\end{aligned}
$$

For large $n, \ln \left(1+\eta_{n}^{-1}\right) \simeq o\left(n^{-2}\right)$ and therefore:

$$
\lim _{n \rightarrow \infty} \ln \eta_{n+1}-a_{1} * \ln \eta_{n}=\frac{2 h}{T},
$$

or

$$
\lim _{n \rightarrow \infty} \frac{\ln \eta_{n}}{n}=\frac{2 h}{T} .
$$

Thus the following equations determine $\eta_{n}$,

$$
\begin{aligned}
& \ln \eta_{1}(x)=\frac{2 \pi J}{T} s(x)+s * \ln \left(1+\eta_{2}(x)\right), \\
& \ln \eta_{n}(x)=s * \ln \left(1+\eta_{n-1}(x)\right)\left(1+\eta_{n+1}(x)\right), \\
& \lim _{n \rightarrow \infty} \frac{\ln \eta_{n}}{n}=\frac{2 h}{T},
\end{aligned}
$$

where

$$
s(x)=\frac{1}{4} \operatorname{sech}\left(\frac{\pi x}{2}\right) .
$$




\section{Some special cases and expansions}

\section{$11.1 J / T \rightarrow 0$ case}

In the limit $J / T \rightarrow 0$ and $h / T \geq 0$ we can expect that $\eta_{n}(x)$ is independent of $x$, because there are no $x$ dependent terms in equations (115), (116 and (117). As $\int d x s(x)=1 / 2$, equations $(115,116,117)$ become

$$
\begin{aligned}
& \eta_{n}^{2}=\left(1+\eta_{n-1}\right)\left(1+\eta_{n+1}\right), \\
& \eta_{1}^{2}=1+\eta_{2}, \lim _{n \rightarrow \infty} \ln \eta_{n} / n=2 h / T .
\end{aligned}
$$

Equation (119) is a difference equation of second order. It is similar to a differential equation of the second order and contains two arbitrary parameters. The general solution of this equation is

$$
\eta_{n}=\left(\frac{a z^{n}-a^{-1} z^{-n}}{z-z^{-1}}\right)^{2}-1 .
$$

Parameters $a$ and $z$ are determined by (120) and we have $a=z, z=\exp (h / T)$ and

$$
\begin{gathered}
\eta_{n}=\left(\frac{\sinh [(n+1) h / T]}{\sinh [h / T]}\right)^{2}-1 \quad \text { for } h>0 \\
\eta_{n}=(n+1)^{2}-1 \quad \text { for } \quad h=0 .
\end{gathered}
$$

Substituting this into (104) we obtain the free energy, magnetization and entropy

$$
\begin{aligned}
& f=-T \ln [2 \cosh h / T], \quad m=2 s_{z}=-\partial f / \partial h=\tanh h / T, \\
& s=-\partial f / \partial T=\ln [2 \cosh (h / T)]-(h / T) \tanh (h / T) .
\end{aligned}
$$

At $h=0$ the entropy per site is $\ln 2$. This corresponds to the fact that the number of states per site is two.

\subsection{High temperature expansion or small $J$ expansion}

For the XXX chain, we can perform the high temperature expansion of the free energy density from the definition

$$
f / T=-N^{-1} \ln \operatorname{Tr} \exp (-\mathcal{H} / T) .
$$

This is expanded as a power series of $1 / T$. Assume that $\mathcal{H}=\mathcal{H}_{0}+\mathcal{H}_{1}$ where $\mathcal{H}_{0}$ and $\mathcal{H}_{1}$ commute each other. Then the exponential operator of $\mathcal{H}$ can be expanded as follows

$$
\exp (-\mathcal{H} / T)=\exp \left(-\mathcal{H}_{0} / T\right)\left(1-T^{-1} \frac{\mathcal{H}_{1}}{1 !}+T^{-2} \frac{\mathcal{H}_{2}}{2 !}-+\ldots\right) .
$$

Thus,

$$
f / T=-N^{-1} \ln \operatorname{Tr} \exp \left(-\mathcal{H}_{0} / T\right)+\frac{<\mathcal{H}_{1}>}{N T}-\frac{<\mathcal{H}_{1}^{2}>-<\mathcal{H}_{1}>^{2}}{2 ! N T^{2}}
$$




$$
+\frac{<\mathcal{H}_{1}^{3}>-3<\mathcal{H}_{1}>^{2}<\mathcal{H}_{1}>+2<\mathcal{H}_{1}>^{3}}{3 ! N T^{3}}-+\ldots
$$

where

$$
<X>\equiv \frac{\operatorname{Tr} \exp \left(-\mathcal{H}_{0} / T\right) X}{\operatorname{Tr} \exp \left(-\mathcal{H}_{0} / T\right)}
$$

In the beginning of 1930's only the ferromagnetic model was considered. If we set

$$
\mathcal{H}_{0}=-2 h \sum_{l=1} S_{l}^{z}, \quad \mathcal{H}_{1}=-J \sum_{l=1}^{N} S_{l}^{x} S_{l+1}^{x}+S_{l}^{y} S_{l+1}^{y}+S_{l}^{z} S_{l+1}^{z},
$$

for the Hamiltonian (46), we obtain the $J / T$ expansion of free energy at fixed $h / T$

$$
\begin{aligned}
& f / T=-\ln (2 \cosh h / T)-\frac{J}{4 T} \tanh ^{2}(h / T) \\
& -\frac{J^{2}}{32 T^{2}}\left(3+2 \tanh ^{2}(h / T)-3 \tanh ^{4}(h / T)\right)+O\left((J / T)^{3}\right) .
\end{aligned}
$$

The calculation to higher orders can be done by the use of linked cluster expansion. Higher order terms are polynomials of $\tanh (h / T)$.

Apparently the expression of the free energy in (123) coincides with the first term of the above expansion. Writing $\ln \left(\eta_{n}+1\right)$ as the expansion

$$
\begin{aligned}
& \ln \left(\eta_{n}(x)+1\right)=\ln \left[\frac{1}{\alpha_{n}-1}\right]+\sum_{l=1}^{\infty} f_{n}^{(l)}\left[\frac{J}{T}\right]^{l}, \\
& \alpha_{n} \equiv \frac{\sinh ^{2}(h(n+1) / T)}{\sinh (h n / T) \sinh (h(n+2) / T)},
\end{aligned}
$$

we obtain an expansion of $\ln \eta_{n}(x)$

$$
\begin{aligned}
& \ln \eta_{n}(x)=\ln \frac{\alpha_{n}}{\alpha_{n}-1}+\left[\frac{J}{T}\right] \alpha_{n} f_{n}^{(1)} \\
& +\left[\frac{J}{T}\right]^{2}\left(\alpha_{n} f_{n}^{(2)}+\left(\alpha_{n}-\alpha_{n}^{2}\right) \frac{\left(f_{n}^{(1)}\right)^{2}}{2}\right)+O\left(\left[\frac{J}{T}\right]^{3}\right) .
\end{aligned}
$$

Substituting these expansions into $(115,116,117,118)$ and taking first order terms in $J / T$, linear integral equations for $f_{n}^{(1)}(x)$ are obtained

$$
\begin{aligned}
& \alpha_{1} f_{1}^{(1)}(x)-s * f_{2}^{(1)}(x)=2 \pi s(x), \\
& \alpha_{n} f_{n}^{(1)}(x)-s *\left(f_{n-1}^{(1)}(x)+f_{n+1}^{(1)}(x)\right)=0, \\
& \lim _{n \rightarrow \infty} \frac{\alpha_{n} f_{n}(x)}{n}=0 .
\end{aligned}
$$


The r.h.s. of these equations are inhomogeneous terms of the integral equations. The Fourier transform of these equations are

$$
\left(e^{|\omega|}+e^{-|\omega|}\right) \alpha_{n} \tilde{f}_{n}^{(1)}(\omega)=\tilde{f}_{n-1}^{(1)}(\omega)+\tilde{f}_{n+1}^{(1)}(\omega) .
$$

The general solution of this difference equation is

$$
\begin{aligned}
& \tilde{f}_{n}^{(1)}(\omega)= \\
& A(\omega)\left[\frac{\sinh ((n+2) h / T)}{\sinh ((n+1) h / T)} e^{-n|\omega|}-\frac{\sinh (n h / T)}{\sinh ((n+1) h / T)} e^{-(n+2)|\omega|}\right] \\
& +B(\omega)\left[\frac{\sinh ((n+2) h / T)}{\sinh ((n+1) h / T)} e^{n|\omega|}-\frac{\sinh (n h / T)}{\sinh ((n+1) h / T)} e^{(n+2)|\omega|}\right]
\end{aligned}
$$

From the boundary conditions we have

$$
A(\omega)=\frac{\pi}{\cosh h / T}, \quad B(\omega)=0 .
$$

Thus

$$
\begin{aligned}
& \tilde{f}_{1}^{(1)}(\omega)=\frac{\pi}{\cosh (h / T)}\left[\frac{\sinh 3 h / T}{\sinh 2 h / T} e^{-|\omega|}-\frac{\sinh h / T}{\sinh 2 h / T} e^{-3|\omega|}\right] \\
& f_{1}^{(1)}(x)=\frac{\pi}{\cosh (h / T)}\left[\frac{\sinh 3 h / T}{\sinh 2 h / T} a_{1}(x)-\frac{\sinh h / T}{\sinh 2 h / T} a_{3}(x)\right] .
\end{aligned}
$$

Substituting this into (104) we obtain the second term of the $J / T$ expansion (129). The higher order terms can be calculated by solving the linear integral equations for $f_{n}^{(2)}, f_{n}^{(3)}, \ldots$. The equations are similar to $(132,133,134)$ except for the inhomogeneous terms, which are given by lower order $f_{n}^{(l)}$.

\subsection{Low temperature limit}

At low temperature, $\ln \eta_{n}$ diverges as $1 / T$. So we should define the following functions

$$
\epsilon_{n}(x)=T \ln \eta_{n}(x) .
$$

The integral equations become

$$
\begin{aligned}
& \epsilon_{1}(x)=2 \pi J s(x)+s * T \ln \left(1+\exp \left(\frac{\epsilon_{2}(x)}{T}\right)\right), \\
& \epsilon_{n}(x)=s * T \ln \left(1+\exp \left(\frac{\epsilon_{n-1}(x)}{T}\right)\right)\left(1+\exp \left(\frac{\epsilon_{n+1}(x)}{T}\right)\right), \\
& \lim _{n \rightarrow \infty} \frac{\epsilon_{n}(x)}{n}=2 h .
\end{aligned}
$$


The free energy expression becomes

$$
\begin{aligned}
f & =-\left(\frac{J}{4}+h\right)-T \sum_{n=1}^{\infty} \int a_{n}(x) \ln \left(1+\exp \left(-\epsilon_{n}(x) / T\right) d x\right. \\
& =J\left(\ln 2-\frac{1}{4}\right)-T \int s(x) \ln \left(1+\exp \left(\epsilon_{1}(x) / T\right)\right) d x
\end{aligned}
$$

The $T=0$ limit of these equations is

$$
\begin{gathered}
\epsilon_{1}(x)=2 \pi J s(x)+s * \epsilon_{2}^{+}(x), \\
\epsilon_{n}(x)=s *\left(\epsilon_{n-1}^{+}(x)+\epsilon_{n+1}^{+}(x)\right), \\
\lim _{n \rightarrow \infty} \frac{\epsilon_{n}(x)}{n}=2 h, \\
f=-\left(\frac{J}{4}+h\right)+\sum_{n=1}^{\infty} \int a_{n}(x) \epsilon_{n}^{-}(x) d x=J\left(\ln 2-\frac{1}{4}\right)-\int s(x) \epsilon_{1}^{+}(x) d x, \\
\epsilon_{n}^{+}(x) \equiv \begin{cases}\epsilon_{n}(x), & \text { for } \epsilon_{n}(x) \geq 0, \\
0, & \text { for } \epsilon_{n}(x)<0, .\end{cases} \\
\epsilon_{n}^{-}(x) \equiv \begin{cases}0, & \text { for } \epsilon_{n}(x) \geq 0, \\
\epsilon_{n}(x), & \text { for } \epsilon_{n}(x)<0 .\end{cases}
\end{gathered}
$$

In the ferromagnetic case $J>0$ we have

$$
\epsilon_{n}(x)=\epsilon_{n}^{+}(x)=2 \pi J a_{n}(x)+2 h n, \quad n=1,2, \ldots,
$$

and therefore $f=-\left(\frac{J}{4}+h\right)$. This is the ground state energy of the ferromagnetic case.

In the antiferromagnetic case $J<0$ we have

$$
\epsilon_{n}(x)=\epsilon_{n}^{+}(x)=a_{n-1} * \epsilon_{1}^{+}(x)+2(n-1) h, \quad n=2,3, \ldots
$$

The equation which determines $\epsilon_{1}$ is

$$
\epsilon_{1}(x)=-2 \pi|J| s(x)+h+\int_{|y|>B} R(x-y) \epsilon_{1}(y) d y, \quad \epsilon_{1}( \pm B)=0 .
$$

In the limit of $h \rightarrow 0 B$ becomes infinite. We have $\epsilon_{1}(x)=-2 \pi|J| s(x)$ and $f=J\left(\ln 2-\frac{1}{4}\right)$. 


\subsection{Fugacity expansion}

In the case of very large $h$ the free energy can be expanded as a power series of $z=\exp (-h / T), z$ is the called the fugacity. From equation (94) and (99) we have expansions of $\eta_{n}^{-1}$ as follows

$$
\eta_{n}^{-1}=z^{2 n} \exp \left(-\frac{2 \pi J}{T} a_{n}(x)\right) \exp \left[-T_{n m} *\left(\eta_{m}^{-1}-\frac{1}{2} \eta_{m}^{-2}+-\ldots\right)\right] .
$$

The expansion of $\eta_{1}^{-1}$ and $\eta_{2}^{-1}$ up to $z^{4}$ is

$$
\begin{aligned}
& \eta_{1}^{-1}=z^{2} \exp \left(-\frac{2 \pi J}{T} a_{1}(x)\right)\left(1-z^{2} \int a_{2}(x-y) \exp \left(-\frac{2 \pi J}{T} a_{1}(y)\right) d y\right)+O\left(z^{6}\right) \\
& \eta_{2}^{-1}=z^{4} \exp \left(-\frac{2 \pi J}{T} a_{2}(x)\right)+O\left(z^{6}\right) .
\end{aligned}
$$

As $\eta_{n}^{-1}$ becomes small, (102) is more convenient than (104)

$$
f=-\frac{J}{4}-h-T \sum_{n=1}^{\infty} \int d x a_{n}(x)\left(\eta_{n}^{-1}-\frac{1}{2} \eta_{n}^{-2}+-\ldots\right) .
$$

Substituting (148), we obtain

$$
\begin{aligned}
& f=-\frac{J}{4}-h-z^{2} T \int a_{1}(x) \exp \left(-\frac{2 \pi J}{T} a_{1}(x)\right) d x \\
& -z^{4} T\left\{\int a_{2}(x) \exp \left(-\frac{2 \pi J}{T} a_{2}(x)\right)-\frac{1}{2} a_{1}(x) \exp \left(-\frac{4 \pi J}{T} a_{1}(x)\right) d x\right. \\
& \left.-\int d x \int d y a_{1}(x) \exp \left[-\frac{2 \pi J}{T}\left(a_{1}(x)+a_{1}(y)\right)\right] a_{2}(x-y)\right\}+O\left(z^{6}\right) .
\end{aligned}
$$

Putting $x=\tan u, y=\tan v$,

$$
\begin{aligned}
& \left(f+\frac{J}{4}+h\right) / T \\
& =z^{2} e^{-K} I_{0}(K)+z^{4}\left\{-\frac{1}{2} e^{-2 K} I_{0}(2 K)+e^{-K / 2} I_{0}(K / 2)\right. \\
& \left.-\frac{1}{\pi^{2}} \int_{0}^{\pi} \int_{0}^{\pi} \frac{e^{2 K\left(1-\cos \omega_{1} \cos \omega_{2}\right)}\left(1-\cos \omega_{1} \cos \omega_{2}\right)}{1-2 \cos \omega_{1} \cos \omega_{2}+\cos ^{2} \omega_{1}} d \omega_{1} d \omega_{2}\right\} \\
& +O\left(z^{6}\right),
\end{aligned}
$$

where $K \equiv J / T, \omega_{1}=\pi+u+v, \omega_{2}=u-v$ and $I_{0}(x)$ is modified Bessel function. This result is the same as that of Katsura (Katsura (1965)).

The strings are stable in a chain of infinite length in the case of very few down spins. Some counter examples of string assumption are found in some special limit. Nevertheless the thermodynamic Bethe ansatz equation seems to give the exact free energy in the case where the density of down-spins is comparable to that of up-spins. This non-linear integral equation contains an infinite number of unknown functions. To solve this equation one needs to do numerical calculations by computer. 


\section{Thermodynamics of the $\mathrm{XXZ}$ model}

\section{Symmetry of the Hamiltonian}

In this section we consider the following Hamiltonian

$$
\begin{aligned}
& \mathcal{H}(J, \Delta, h)=-J \sum_{l=1}^{N} S_{l}^{x} S_{l+1}^{x}+S_{l}^{y} S_{l+1}^{y}+\Delta S_{l}^{z} S_{l+1}^{z}-2 h \sum_{l=1} S_{l}^{z}, \\
& h \geq 0, \quad \mathbf{S}_{N+1} \equiv \mathbf{S}_{1} .
\end{aligned}
$$

This Hamiltonian contains an additional parameter $\Delta$. The case $\Delta=0$ is called the XY model, which can be mapped to non-interacting fermions making it possible to calculate many physical quantities (Lieb Schultz and Mattis (1961), Katsura (1962)). The case $\Delta=1$ is the XXX model and was treated in the previous chapter. The limit of very large $\Delta$ is the Ising model. The generalization of Bethe's method to $\Delta \neq 1$ was done by Orbach and Walker (Orbach 1958 Walker 1959). Yang and Yang investigated the ground state of this model in detail (Yang and Yang (1966)). Bonner and Fisher investigated this model using the diagonalization method up to $N=12$ (Bonner and Fisher (1964)). In this Hamiltonian the magnetic field is applied in the $\mathrm{z}$-direction. For a magnetic field in a different direction, the exact solution is not known.

Let us consider the following unitary transformation:

$$
\mathcal{H}(J, \Delta, h)=U_{1} \mathcal{H}(J, \Delta,-h) U_{1}^{-1}, \quad U_{1} \equiv \prod_{l=1}^{\infty} 2 S_{l}^{x}=U_{1}^{-1}
$$

By this unitary transformation $S_{\text {total }}^{z}$ changes its sign and we can treat the $N \geq M>N / 2$ case. In the case of even $N$ we can show that

$$
\mathcal{H}(-J,-\Delta, h)=U_{2} \mathcal{H}(J, \Delta, h) U_{2}^{-1}, \quad U_{2} \equiv \prod_{l=\text { even }} 2 S_{l}^{z}=U_{2}^{-1}
$$

By this unitary transformation $S_{l}^{x}, S_{l}^{y}, S_{l}^{z}$ change to $-S_{l}^{x},-S_{l}^{y}, S_{l}^{z}$ at $l=$ even. 


\section{Bethe ansatz wave function}

Consider the state where all spins are up and the total $S^{z}$ is $N / 2$

$$
\mathcal{H}\left|0>=E_{0}\right| 0>, \quad E_{0}=-J \Delta N / 4-N h .
$$

Writing a general state $|\Psi\rangle$ in terms of a wave function $f$, as in equation (48), the eigenvalue condition can be expressed as

$$
\begin{aligned}
& 0=-\frac{J}{2} \sum_{j}\left(1-\delta_{n_{j}+1, n_{j+1}}\right)\left\{f\left(n_{1}, ., n_{j}+1, n_{j+1}, ., n_{M}\right)\right. \\
& \left.+f\left(n_{1}, ., n_{j}, n_{j+1}-1, ., n_{m}\right)\right\} \\
& +\left\{E_{0}-E+(J \Delta+2 h) M-J \Delta \sum_{j} \delta_{n_{j}+1, n_{j+1}}\right\} f\left(n_{1}, n_{2}, ., n_{M}\right) .
\end{aligned}
$$

For a wave function of the type eq. (50) to be the eigenstate, set $E$ and $A(P)$ to be

$$
\begin{aligned}
& E=E_{0}+\sum_{j=1}^{M}\left[J\left(\Delta-\cos k_{j}\right)+h\right], \\
& 0=A(P)\left(e^{i k_{P j}}+e^{-k_{P(j+1)}}-2 \Delta\right) e^{k_{P(j+1)}} \\
& +A(P(j, j+1))\left(e^{i k_{P(j+1)}}+e^{-k_{P j}}-2 \Delta\right) e^{k_{P j}}, \\
& A(P)=\epsilon(P) \prod_{l<j}\left(e^{i\left(k_{P l}+k_{P j}\right)}+1-2 \Delta e^{i k_{P l}}\right) .
\end{aligned}
$$

The periodic boundary condition is as follows,

$$
\begin{array}{r}
\exp \left(i k_{j} N\right)=(-1)^{M-1} \prod_{l \neq j} \frac{\exp \left[i\left(k_{j}+k_{l}\right)\right]+1-2 \Delta \exp \left(i k_{j}\right)}{\exp \left[i\left(k_{j}+k_{l}\right)\right]+1-2 \Delta \exp \left(i k_{l}\right)} \\
j=1,2, \ldots, M .
\end{array}
$$

This is a set of complicated coupled equations for $M$ unknowns. If we have a solution of this set, we have one eigenstate and its energy eigenvalue and total momentum. If we set rapidity parameters $x_{j}$ as $\cot \left(k_{j} / 2\right)$

$$
\exp \left(i k_{j}\right)=\left(\frac{x_{j}+i}{x_{j}-i}\right),
$$

the phase factor

$$
\frac{\exp \left[i\left(k_{j}+k_{l}\right)\right]+1-2 \Delta \exp \left(i k_{j}\right)}{\exp \left[i\left(k_{j}+k_{l}\right)\right]+1-2 \Delta \exp \left(i k_{l}\right)}
$$


cannot be written as a function of $x_{j}-x_{l}$ except in the case $\Delta=1$. This is not convenient. If we set

$$
\exp \left(i k_{j}\right)=\frac{\sin \frac{\phi}{2}\left(x_{j}+i\right)}{\sin \frac{\phi}{2}\left(x_{j}-i\right)}
$$

in place of (160), the phase factor becomes

$$
\frac{\cos \frac{\phi}{2}\left(x_{j}+x_{l}\right)(\cosh \phi-\Delta)+\left(\Delta \cos \frac{\phi}{2}\left(x_{j}-x_{l}+2 i\right)-\cos \frac{\phi}{2}\left(x_{j}-x_{l}\right)\right)}{\cos \frac{\phi}{2}\left(x_{j}+x_{l}\right)(\cosh \phi-\Delta)+\left(\Delta \cos \frac{\phi}{2}\left(x_{l}-x_{j}+2 i\right)-\cos \frac{\phi}{2}\left(x_{l}-x_{j}\right)\right)} .
$$

At $\cosh \phi-\Delta=0$, this phase factor becomes a function of $x_{j}-x_{l}$ and independent of $x_{j}+x_{l}$. It is written as

$$
\sin \frac{\phi}{2}\left(x_{j}-x_{l}+2 i\right) / \sin \frac{\phi}{2}\left(x_{j}-x_{l}-2 i\right) .
$$

Then for $\Delta>1$ (159) becomes

$$
\begin{array}{r}
\left(\frac{\sin \frac{\phi}{2}\left(x_{j}+i\right)}{\sin \frac{\phi}{2}\left(x_{j}-i\right)}\right)^{N}=\prod_{l \neq j} \frac{\sin \frac{\phi}{2}\left(x_{j}-x_{l}+2 i\right)}{\sin \frac{\phi}{2}\left(x_{j}-x_{l}-2 i\right)}, \\
\phi=\cosh ^{-1} \Delta, \quad \phi>0 .
\end{array}
$$

For $\Delta<-1$ we set

$$
\exp \left(i k_{j}\right)=-\frac{\sin \frac{\phi}{2}\left(x_{j}+i\right)}{\sin \frac{\phi}{2}\left(x_{j}-i\right)}
$$

Equation (159) becomes

$$
\begin{aligned}
\left(\frac{\sin \frac{\phi}{2}\left(x_{j}+i\right)}{\sin \frac{\phi}{2}\left(x_{j}-i\right)}\right)^{N} & =\prod_{l \neq j} \frac{\sin \frac{\phi}{2}\left(x_{j}-x_{l}+2 i\right)}{\sin \frac{\phi}{2}\left(x_{j}-x_{l}-2 i\right)}, \\
\phi & =\cosh ^{-1}(-\Delta), \quad \phi>0 .
\end{aligned}
$$

In the case $1>\Delta>-1$ we set

$$
\exp \left(i k_{j}\right)=-\frac{\sinh \frac{\gamma}{2}\left(x_{j}+i\right)}{\sinh \frac{\gamma}{2}\left(x_{j}-i\right)}
$$

yielding the Bethe ansatz equations

$$
\begin{aligned}
& \left(\frac{\sinh \frac{\gamma}{2}\left(x_{j}+i\right)}{\sinh \frac{\gamma}{2}\left(x_{j}-i\right)}\right)^{N}=\prod_{l \neq j} \frac{\sinh \frac{\gamma}{2}\left(x_{j}-x_{l}+2 i\right)}{\sinh \frac{\gamma}{2}\left(x_{j}-x_{l}-2 i\right)}, \\
& \gamma=\cos ^{-1}(-\Delta), \quad \pi>\gamma>0 .
\end{aligned}
$$




\section{String solutions at $\Delta>1$}

By the transformation (161) the wave function and eigenvalue are written as follows,

$$
\begin{aligned}
& f\left(n_{1}, n_{2}, \ldots, n_{M}\right) \\
&= \sum_{P} \epsilon(P) \prod_{j<l} \sin \frac{\phi}{2}\left(x_{P j}-x_{P l}+2 i\right) \prod_{j=1}^{M}\left(\frac{\sin \frac{\phi}{2}\left(x_{P j}+i\right)}{\sin \frac{\phi}{2}\left(x_{P j}-i\right)}\right)^{n_{j}}, \\
& E=E_{0}+\sum_{j=1}^{M}\left(2 h+\frac{J \sinh ^{2} \phi}{\cosh \phi-\cos \phi x_{j}}\right), \quad K=\sum_{j} 2 \cot ^{-1} \frac{\tan \left(\phi x_{j} / 2\right)}{\tanh (\phi / 2)} .
\end{aligned}
$$

The following string solutions are possible for complex $x_{j}$ 's for the $N=\infty$ case from the normalizability of the wave function,

$$
x_{j}=\alpha+(M+1-2 j) i,
$$

where $\alpha$ is a real number at $-Q<\alpha \leq Q, Q \equiv \pi / \phi$. The total momentum and energy is given by

$$
K=2 \cot ^{-1} \frac{\tan (\phi \alpha / 2)}{\tanh (M \phi / 2)}, \quad E=E_{0}+\frac{J \sinh \phi \sinh M \phi}{\cosh M \phi-\cos \phi \alpha}+2 M h .
$$

Thus the dispersion is

$$
E=E_{0}+2 M h+J \sinh \phi\left[\frac{\cosh M \phi-\cos K}{\sinh M \phi}\right] .
$$

This excitation energy gives $(71)$ in the limit $\Delta \rightarrow 1$. In the limit of large $\Delta$ the energy is $J \Delta+2 M h$. This is $M$ successive down spins in a sea of up spins. The lowest energy state at $\Delta>1$ with $M$ down spins is the $M$ string state given by (171) with zero total momentum. So the energy of the ground state for $\Delta>1$ is

$$
E=-\frac{J N \Delta}{4}-(N-2 M) h+J \sinh \phi \tanh \frac{M \phi}{2} .
$$

\section{Thermodynamic equations for the XXZ model for $\Delta>1$}

Gaudin derived a set of thermodynamic Bethe ansatz equations at $\Delta>1$

(Gaudin (1971)). The wave function for $M$ down spins in the infinite lattice is

$$
\begin{aligned}
& f\left(n_{1}, n_{2}, \ldots, n_{M}\right)=\left(z_{1} z_{2} . . z_{M}\right)^{n_{1}} \sum_{P} A(P) \prod_{j=2}^{M}\left(\prod_{l=j}^{M} z_{P l}\right)^{n_{j+1}-n_{j}}, \\
& z_{j}=e^{i k_{j}}=\left(\frac{\sin \frac{\phi}{2}\left(x_{j}+i\right)}{\sin \frac{\phi}{2}\left(x_{j}-i\right)}\right) .
\end{aligned}
$$


This corresponds to (73). From the normalizability condition of the wave function we have

$$
\begin{array}{r}
A(I) \neq 0, A(P \neq I)=0, \quad\left|z_{1} z_{2} \ldots z_{M}\right|=1 \\
\left|\prod_{l=j+1}^{M} z_{l}\right| \leq 1, \quad j=1,2,3, \ldots, M-1 .
\end{array}
$$

These conditions are satisfied only if

$$
x_{j}=\alpha+(M+1-2 j) i, \quad j=1,2, \ldots, M, \quad Q \geq \alpha>-Q .
$$

We can show that

$$
\left|\prod_{l=j}^{M} z_{l}\right|=\left|\frac{\sin \frac{\phi}{2}(\alpha+i(M-2 j))}{\sin \frac{\phi}{2}(\alpha-i M)}\right|=\sqrt{\frac{\cosh \phi(M-2 j)-\cos \phi \alpha}{\cosh \phi n-\cos \phi \alpha}} \leq 1,
$$

for arbitrary $M$. Thus a string with arbitrary length is possible for the XXZ chain at $|\Delta|>1$. In the case $|\Delta|<1$ the string condition is more complicated than the case $|\Delta| \geq 1$. From (162) we have the following equation corresponding to $(80)$,

$$
e_{n}^{N}\left(x_{\alpha}^{n}\right)=\prod_{j=1}^{n} e^{N}\left(x_{\alpha}^{n, j}\right)=\prod_{(m, \beta) \neq(n, \alpha)} E_{n m}\left(x_{\alpha}^{n}-x_{\beta}^{m}\right) .
$$

Here

$$
\begin{gathered}
e_{n}(x)=\frac{\sin \frac{\phi}{2}(x+i n)}{\sin \frac{\phi}{2}(x-i n)}, \\
E_{n m}(x) \equiv\left\{\begin{array}{r}
e_{|n-m|}(x) e_{|n-m|+2}^{2}(x) e_{|n-m|+4}^{2}(x) \ldots e_{n+m-2}^{2}(x) e_{n+m}(x) \\
\text { for } n \neq m, \\
e_{2}^{2}(x) e_{4}^{2}(x) \ldots e_{2 n-2}^{2}(x) e_{2 n}(x) \text { for } n=m .
\end{array}\right.
\end{gathered}
$$

$x_{\alpha}^{n}$ is the real part of $\alpha$-th string in the strings of length $n$. The logarithm of (176) is

$$
N \theta_{n}\left(x_{\alpha}^{n}\right)=2 \pi I_{\alpha}^{n}+\sum_{(m, \beta) \neq(n, \alpha)} \Theta_{n m}\left(x_{\alpha}^{n}-x_{\beta}^{m}\right),
$$

where

$$
\theta_{n}(x)=2 \tan ^{-1}\left(\frac{\tan \frac{x \phi}{2}}{\tanh \frac{n \phi}{2}}\right)+2 \pi\left[\frac{x+Q}{2 Q}\right]
$$

and

$$
\Theta_{n m}(x) \equiv\left\{\begin{array}{l}
\theta_{|n-m|}(x)+2 \theta_{|n-m|+2}(x)+\ldots+2 \theta_{n+m-2}(x)+\theta_{n+m}(x) \\
\text { for } n \neq m, \\
2 \theta_{2}(x)+2 \theta_{4}(x)+\ldots+2 \theta_{2 n-2}(x)+\theta_{2 n}(x) \text { for } n=m .
\end{array}\right.
$$


The function $\theta_{n}(x)$ is a quasi periodic function which satisfies

$$
\theta_{n}(x+2 j Q)=\theta_{n}(x)+2 \pi j, \quad j=\text { integer } .
$$

We consider the energy of general eigenstates which is given by the set of quantum numbers $\left\{I_{\alpha}^{n}\right\}$,

$$
E\left(\left\{I_{\alpha}^{n}\right\}\right)=N\left(-h-\frac{J \Delta}{4}\right)+\sum_{n, \alpha}\left(\frac{2 \pi J \sinh \phi}{\phi} \mathbf{a}_{n}\left(x_{\alpha}^{n}\right)+2 h n\right),
$$

where

$$
\mathbf{a}_{n}(x)=\frac{1}{2 \pi} \frac{\phi \sinh n \phi}{\cosh n \phi-\cos \phi x} .
$$

The partition function of the XXZ model is as follows,

$$
\mathcal{Z}=\sum_{M=0}^{[N / 2]}\left(1+\left(1-\delta_{N, 2 M}\right) \exp -\frac{(N-2 M) h}{T}\right) \sum_{\left\{I_{\alpha}^{n}\right\}} \exp \left[-T^{-1} E\left(\left\{I_{\alpha}^{n}\right\}\right)\right] .
$$

Corresponding to (88) we define the following functions,

$$
h_{n}(x) \equiv \theta_{n}(x)-N^{-1} \sum_{(m, \alpha)} \Theta_{n m}\left(x-x_{\alpha}^{m}\right) .
$$

Using this function we can determine the position of holes for n-strings. We define the distribution functions of particles and holes of n-strings as $\rho_{n}(x)$ and $\rho_{n}^{h}(x)$. By the equation (179) we have the conditions for these two kinds of functions

$$
\mathbf{a}_{n}(x)=\rho_{n}(x)+\rho_{n}^{h}(x)+\sum_{m} \mathbf{T}_{n m} * \rho_{m}(x) .
$$

Here $\mathbf{a}_{n}$ was defined in (182) and

$$
\mathbf{T}_{n m}(x) \equiv\left\{\begin{array}{c}
\mathbf{a}_{|n-m|}(x)+2 \mathbf{a}_{|n-m|+2}(x)+2 \mathbf{a}_{|n-m|+4}(x)+\ldots \\
+2 \mathbf{a}_{n+m-2}(x)+\mathbf{a}_{n+m}(x) \text { for } n \neq m, \\
2 \mathbf{a}_{2}(x)+2 \mathbf{a}_{4}(x)+\ldots+2 \mathbf{a}_{2 n-2}(x)+\mathbf{a}_{2 n}(x) \text { for } n=m .
\end{array}\right.
$$

Here the meaning of convolution of two periodic functions $\mathbf{a}$ and $\mathbf{b}$ with periodicity $2 Q$ is redefined

$$
\mathbf{a} * \mathbf{b}(x) \equiv \int_{-Q}^{Q} \mathbf{a}(x-y) \mathbf{b}(y) d y,
$$

The energy per site is

$$
\begin{gathered}
e=-\left(\frac{J \Delta}{4}+h\right)+\sum_{n=1}^{\infty} \int_{-Q}^{Q} \mathbf{g}_{n}(x) \rho_{n}(x) d x \\
\mathbf{g}_{n}(x) \equiv \frac{2 \pi J \sinh \phi}{\phi} \mathbf{a}_{n}(x)+2 n h .
\end{gathered}
$$


The entropy per site $s$ is

$$
s=\sum_{n=1}^{\infty} \int_{-Q}^{Q} \rho_{n}(x) \ln \left(1+\frac{\rho_{n}^{h}(x)}{\rho_{n}(x)}\right)+\rho_{n}^{h}(x) \ln \left(1+\frac{\rho_{n}(x)}{\rho_{n}^{h}(x)}\right) d x .
$$

The condition of minimizing the free energy $e-T s$ yields equations for $\eta_{n}(x) \equiv$ $\rho_{n}^{h}(x) / \rho_{n}(x)$,

$$
\ln \eta_{n}(x)=\frac{\mathbf{g}_{n}(x)}{T}+\sum_{m=1}^{\infty} \mathbf{T}_{n m} * \ln \left(1+\eta_{m}^{-1}(x)\right) .
$$

This set of equations is equivalent to the following one

$$
\begin{aligned}
& \ln \eta_{1}(x)=\frac{2 \pi J \sinh \phi}{T \phi} \mathbf{s}(x)+\mathbf{s} * \ln \left(1+\eta_{2}(x)\right), \\
& \ln \eta_{n}(x)=\mathbf{s} * \ln \left(1+\eta_{n-1}(x)\right)\left(1+\eta_{n+1}(x)\right), \\
& \lim _{n \rightarrow \infty} \frac{\ln \eta_{n}}{n}=\frac{2 h}{T},
\end{aligned}
$$

where

$$
\mathbf{s}(x)=\frac{1}{4} \sum_{n=-\infty}^{\infty} \operatorname{sech}\left(\frac{\pi(x-2 n Q)}{2}\right) .
$$

The free energy per site is

$$
f=-\left(\frac{J \Delta}{4}+h\right)-T \sum_{n=1}^{\infty} \int_{-Q}^{Q} \mathbf{a}_{n}(x) \ln \left(1+\eta_{n}^{-1}(x)\right) d x .
$$

Corresponding to (104) we have another expression for the free energy,

$$
f=J\left[\frac{2 \pi \sinh \phi}{\phi} \int_{-Q}^{Q} \mathbf{a}_{1}(x) \mathbf{s}(x) d x-\frac{\Delta}{4}\right]-T \int_{-Q}^{Q} \mathbf{s}(x) \ln \left(1+\eta_{1}(x)\right) d x .
$$

(115-117) and (191-193) have the almost same structure. These equations are called Gaudin-Takahashi equation (Gaudin (1971), Takahashi (1971a)).

\section{Theory for $|\Delta|<1 \mathrm{XXZ}$ model}

\subsection{String solution of an infinite XXZ model with $|\Delta|<1$}

The shapes of strings for $|\Delta|<1$ are quite different from those at $|\Delta| \geq 1$. Takahashi and Suzuki proposed a condition of the strings and constructed thermodynamic integral equations (Takahashi and Suzuki (1972)). Later Hida, Fowler and Zotos derived these conditions from the normalizability condition of the string wave function for an infinite chain (Hida (1981), Fowler and Zotos (1981)). For 
$|\Delta|<1$ there are two kinds of strings, one of which has the center on the real axis and the other is centered on the $p_{0} i$ axis,

$$
\begin{aligned}
& x_{j}=\alpha+(n+1-2 j) i, \quad j=1,2, \ldots, n, \\
& x_{j}=\alpha+(n+1-2 j) i+p_{0} i, \quad j=1,2, \ldots, n .
\end{aligned}
$$

We designate that the string of the former type has parity $v=1$ and that the latter has parity $v=-1$. Applying the normalizability condition of the form (197) yields

$$
1>\left|\prod_{l=j+1}^{n} z_{l}\right|=\left|\frac{\sinh \frac{\gamma}{2}(\alpha+i(n-2 j))}{\sinh \frac{\gamma}{2}(\alpha-i n)}\right|=\sqrt{\frac{\cosh \gamma \alpha-\cos \gamma(n-2 j)}{\cosh \gamma \alpha-\cos \gamma n}} .
$$

Thus $\cos \gamma n<\cos \gamma(n-2 j)$ for $j=1,2,3, \ldots, n-1$. For (198) we have

$$
1>\left|\prod_{l=j+1}^{n} z_{l}\right|=\left|\frac{\cosh \frac{\gamma}{2}(\alpha+i(n-2 j))}{\cosh \frac{\gamma}{2}(\alpha-i n)}\right|=\sqrt{\frac{\cosh \gamma \alpha+\cos \gamma(n-2 j)}{\cosh \gamma \alpha+\cos \gamma n}},
$$

and therefore $\cos \gamma n>\cos \gamma(n-2 j)$ for $j=1,2,3, \ldots, n-1$. Then from the normalizability condition we get

$$
\begin{aligned}
& 0<v(\cos ((n-2 j) \gamma)-\cos (n \gamma)) \\
& =2 v \sin ((n-j) \gamma) \sin (j \gamma), \text { for } j=1,2, \ldots, n-1 .
\end{aligned}
$$

This is equivalent to

$$
\begin{array}{r}
(-1)^{\left[\frac{(n-j) \gamma}{\pi}\right]+\left[\frac{j \gamma}{\pi}\right]}=v, \text { for } j=1,2, \ldots, n-1, \\
\frac{j \gamma}{\pi} \neq\left[\frac{j \gamma}{\pi}\right], \text { for } j=1,2, \ldots, n-1,
\end{array}
$$

where $[x]$ denotes the maximum integer less than or equal to $x$ (Gauss' symbol). For rational $p_{0}=\pi / \gamma,(201)$ is a strong condition. If $p_{0}=n_{1} / n_{2}$, and $n_{1}$ and $n_{2}$ are coprime, the string with length greater than $n_{1}$ cannot satisfy at least one of (201). Thus $n \geq n_{1}+1$ strings are forbidden. Moreover for a $n=n_{1}$ string, the momentum is always 0 or $\pi$. So this string has also no meaning for the thermodynamics. Next we seek the number $n$ and parity $v$ which satisfies (200) within $n<n_{1}$. Equation (200) is equivalent to

$$
\begin{gathered}
{\left[\frac{(n-j) \gamma}{\pi}\right]+\left[\frac{j \gamma}{\pi}\right] \equiv\left[\frac{(n-j-1) \gamma}{\pi}\right]+\left[\frac{(j+1) \gamma}{\pi}\right](\operatorname{Mod} 2), \quad j=1,2, \ldots, n-2,} \\
{\left[\frac{(n-1) \gamma}{\pi}\right] \equiv \frac{1-v}{2}(\operatorname{Mod} 2) .}
\end{gathered}
$$

As $\left[\frac{(n-j) \gamma}{\pi}\right]-\left[\frac{(n-j-1) \gamma}{\pi}\right]$ is 0 or 1 and $\left[\frac{j \gamma}{\pi}\right]-\left[\frac{(j+1) \gamma}{\pi}\right]$ is 0 or -1 , we obtain

$$
\left[\frac{(n-j) \gamma}{\pi}\right]+\left[\frac{j \gamma}{\pi}\right]=\left[\frac{(n-j-1) \gamma}{\pi}\right]+\left[\frac{(j+1) \gamma}{\pi}\right], \quad j=1,2, \ldots, n-2 .
$$


These are strong restrictions on the parity $v$ and the length of the string $n$. The above conditions are equivalent to the following conditions which were given in (Takahashi and Suzuki (1972)). The length $n$ of a string should satisfy

$$
\begin{aligned}
2 \sum_{j=1}^{n-1}[j \gamma / \pi]= & (n-1)[(n-1) \gamma / \pi], \\
& v \sin \{(n-1) \gamma\} \geq 0 .
\end{aligned}
$$

This condition was first introduced under the assumption that these strings form a complete half-filled state (Takahashi and Suzuki (1972)). Later Hida, Fowler and Zotos showed that conditions $(202,203)$ can be rederived from the normalizability conditions of the wave function for $N \rightarrow \infty$ and finite $M$ (Hida (1981), Fowler and Zotos (1981)). For a given value of $\Delta$ (or $\gamma$ ) we can determine the series of $n$ which satisfies the conditions (202) and (203). If $\gamma / \pi$ is a rational number, this series becomes finite and the number of unknown functions is also finite. We consider the $\gamma=\pi / \nu, \nu=$ integer case. In this case $n=1,2, \ldots, \nu$ satisfy (202). For $n=1$ both $v=1$ and -1 are possible. For $n=2,3, \ldots, \nu-1$, only $v=+1$ states are possible. These excitations have the following energy and momentum

$$
\begin{aligned}
& E=-2 J \frac{\sin \gamma \sin (n \gamma)}{v \cosh (\alpha \gamma)-\cos n \gamma}+2 n h, \\
& K=-i \ln \left(-\frac{\sinh \frac{1}{2}(\alpha \gamma+i(1-v) \pi / 2+i n \gamma)}{\sinh \frac{1}{2}(\alpha \gamma+i(1-v) \pi / 2-i n \gamma)}\right) .
\end{aligned}
$$

The energy and momentum have the following relation,

$$
E=-J \sin \gamma \frac{\cos n \gamma-\cos K}{\sin n \gamma}+2 n h .
$$

The momentum is restricted to the region

$$
\begin{gathered}
|K|<\pi-\left(n \gamma-\pi\left[\frac{n \gamma}{\pi}\right]\right) \text { for } v=1, \\
\pi \geq|K|>\pi-\left(n \gamma-\pi\left[\frac{n \gamma}{\pi}\right]\right) \text { for } v=-1 .
\end{gathered}
$$

Then for $n=\nu$, the energy and momentum are always zero. Only one state is obtained from this string solution. So we exclude this $n=\nu$ state from the thermodynamics of this case. So $\nu$ string states $(1,+),(2,+), \ldots,(\nu-1,+),(1,-)$ play important roles. Especially at $\Delta=0, \gamma=\pi / 2, \nu=2$ we have only string states $(1,+),(1,-)$. These are single states at momentum $|K|<\pi / 2$ and $|K|>$ $\pi / 2$.

Next we consider the $\gamma=\pi /\left(\nu_{1}+1 / \nu_{2}\right)$ case. $(1,+),(2,+), \ldots,\left(\nu_{1}-1,+\right)$, $(1,-),\left(1+\nu_{1},+\right),\left(1+2 \nu_{1},-\right), \ldots\left(1+\left(\nu_{2}-1\right) \nu_{1},(-1)^{\nu_{2}-1}\right),\left(\nu_{1},(-1)^{\nu_{2}}\right)$ satisfy conditions $(202,203)$. Thus $\nu_{1}+\nu_{2}$ strings are necessary to describe the thermodynamics of this case. 
For a general rational number between 0 and 1 , we can express it by a continued fraction with length $l$,

$$
\frac{\gamma}{\pi}=\frac{1 \mid}{\mid \nu_{1}}+\frac{1 \mid}{\mid \nu_{2}}+\ldots+\frac{1 \mid}{\mid \nu_{l}}, \quad \nu_{1}, \nu_{2}, \ldots, \nu_{l-1} \geq 1, \quad \nu_{l} \geq 2 .
$$

We define the following series of numbers $y_{-1}, y_{0}, y_{1}, \ldots, y_{l}$ and $m_{0}, m_{1}, \ldots, m_{l}$ as

$$
\begin{aligned}
& y_{-1}=0, \quad y_{0}=1, \quad y_{1}=\nu_{1} \text { and } y_{i}=y_{i-2}+\nu_{i} y_{i-1} \\
& m_{0}=0, \quad m_{i}=\sum_{k=1}^{i} \nu_{k} .
\end{aligned}
$$

The general rule to determine the parity $v$ and length $n$ is as follows

$$
\begin{aligned}
& n_{j}=y_{i-1}+\left(j-m_{i}\right) y_{i}, \quad v_{j}=(-1)^{\left[\left(n_{j}-1\right) / p_{0}\right]} \text { for } m_{i}<j<m_{i+1}, \\
& n_{m_{l}}=y_{l-1}, \quad v_{m_{l}}=(-1)^{l}
\end{aligned}
$$

The number of strings is $m_{l}$. We give examples for some rational numbers in Tables (1), (2). We put $x_{j}^{\alpha}$ as the real part of strings with parity $v_{j}$ and length $n_{j} . \alpha$ takes values from 1 to $M_{j}$. We find the following relations for these series

\begin{tabular}{|c|c|c|c|c|c|c|c|c|c|}
\hline$j$ & $\frac{1}{5}$ & & & $\frac{3}{16}$ & $=$ & $\frac{1}{5+\frac{1}{3}}$ & $\frac{13}{69}$ & $=$ & $\frac{1}{5+\frac{1}{3+\frac{1}{4}}}$ \\
\hline 1 & 1 & + & $\overline{4}$ & 1 & + & $13 / 3$ & 1 & + & $56 / 13$ \\
\hline 2 & 2 & + & 3 & 2 & + & $10 / 3$ & 2 & + & $43 / 13$ \\
\hline 3 & 3 & + & 2 & 3 & + & $7 / 3$ & 3 & + & $30 / 13$ \\
\hline 4 & 4 & + & 1 & 4 & + & $4 / 3$ & 4 & + & $17 / 13$ \\
\hline 5 & 1 & - & -1 & 1 & - & $-3 / 3$ & 1 & - & $-13 / 13$ \\
\hline 6 & & & & 6 & + & $-2 / 3$ & 6 & + & $-9 / 13$ \\
\hline 7 & & & & 11 & - & $-1 / 3$ & 11 & - & $-5 / 13$ \\
\hline 8 & & & & 5 & + & $1 / 3$ & 5 & + & $4 / 13$ \\
\hline 9 & & & & & & & 21 & - & $3 / 13$ \\
\hline 10 & & & & & & & 37 & + & $2 / 13$ \\
\hline 11 & & & & & & & 53 & - & $1 / 13$ \\
\hline 12 & & & & & & & 16 & - & $-1 / 13$ \\
\hline
\end{tabular}
of numbers

$$
\begin{aligned}
& n_{j}=\frac{1}{2}\left[\left(1-2 \delta_{m_{i}, j}\right) n_{j-1}+n_{j+1}\right], \text { for } m_{i} \leq j \leq m_{i+1}-2, \\
& n_{j}=\left(1-2 \delta_{m_{i-1}, j}\right) n_{j-1}+n_{j+1}, \text { for } j=m_{i}-1, \quad i<l \\
& n_{0}=0, \quad n_{m_{l}}+n_{m_{l}-1}=y_{l} .
\end{aligned}
$$

Table 1. Length $n_{j}$, parity $v_{j}$ and $q_{j}$ of strings for some rational values of $\gamma / \pi$ 
Table 2. Length $n_{j}$, parity $v_{j}$ and $q_{j}$ of strings for conjugate values of $\gamma / \pi$ in previous table

\begin{tabular}{|c|c|c|c|c|c|c|c|c|c|}
\hline$j$ & $\frac{4}{5}$ & $=$ & $\frac{1}{1+\frac{1}{4}}$ & $\frac{13}{16}$ & $=$ & $\frac{1}{1+\frac{1}{4+\frac{1}{3}}}$ & $\frac{56}{69}$ & $=$ & $\frac{1}{1+\frac{1}{4+\frac{1}{3+\frac{1}{4}}}}$ \\
\hline 1 & 1 & - & $-4 / 4$ & $\overline{1}$ & - & $-13 / 13$ & 1 & - & $-56 / 56$ \\
\hline 2 & 2 & + & $-3 / 4$ & 2 & + & $-10 / 13$ & 2 & + & $-43 / 56$ \\
\hline 3 & 3 & - & $-2 / 4$ & 3 & - & $-7 / 13$ & 3 & - & $-30 / 56$ \\
\hline 4 & 4 & + & $-1 / 4$ & 4 & + & $-4 / 13$ & 4 & + & $-17 / 56$ \\
\hline 5 & 1 & + & $1 / 4$ & 1 & + & $3 / 13$ & 1 & + & $13 / 56$ \\
\hline 6 & & & & 6 & + & $2 / 13$ & 6 & + & $9 / 56$ \\
\hline 7 & & & & 11 & + & $1 / 13$ & 11 & + & $5 / 56$ \\
\hline 8 & & & & 5 & - & $-1 / 13$ & 5 & - & $-4 / 56$ \\
\hline 9 & & & & & & & 21 & + & $-3 / 56$ \\
\hline 10 & & & & & & & 37 & - & $-2 / 56$ \\
\hline 11 & & & & & & & 53 & + & $-1 / 56$ \\
\hline 12 & & & & & & & 16 & + & $1 / 56$ \\
\hline
\end{tabular}

\subsection{Scattering phase shift among strings}

Corresponding to (80) and (176) we have Bethe ansatz equations for strings as follows

$$
\left\{e_{j}\left(x_{\alpha}^{j}\right)\right\}^{N}=-\prod_{k=1}^{m_{l}} \prod_{\beta=1}^{M_{k}} E_{j, k}\left(x_{\alpha}^{j}-x_{\beta}^{k}\right)
$$

where

$$
\begin{gathered}
e_{j}(x)=g\left(x ; n_{j}, v_{j}\right), \\
E_{j, k}(x)=\left\{\begin{array}{c}
g\left(x ; 2 n_{j}, v_{j} v_{k}\right) \prod_{l=1}^{n_{j}-1} g^{2}\left(x ; 2 l, v_{j} v_{k}\right) \text { for } n_{j}=n_{k}, \\
g\left(x ;\left(n_{j}+n_{k}\right), v_{j} v_{k}\right) g\left(x ;\left|n_{j}-n_{k}\right|, v_{j} v_{k}\right) \\
\times \prod_{l=1}^{M i n\left(n_{j}, n_{k}\right)-1} g^{2}\left(x ;\left|n_{j}-n_{k}\right|+2 l, v_{j} v_{k}\right) \text { for } n_{j} \neq n_{k},
\end{array}\right. \\
g(x ; n,+)=\frac{\sinh \frac{\gamma}{2}(x+i n)}{\sinh \frac{\gamma}{2}(x-i n)}, g(x ; n,-)=-\frac{\cosh \frac{\gamma}{2}(x+i n)}{\cosh \frac{\gamma}{2}(x-i n)} .
\end{gathered}
$$

The logarithm of (210) yields

$$
N \theta_{j}\left(x_{\alpha}^{j}\right)=2 \pi I_{\alpha}^{j}+\sum_{k=1}^{m_{l}} \sum_{\beta=1}^{M_{k}} \Theta_{j, k}\left(x_{\alpha}^{j}-x_{\beta}^{k}\right), \quad \alpha=1,2, \ldots, M_{j},
$$

where

$$
\begin{array}{r}
\theta_{j}(x)=f\left(x ; n_{j}, v_{j}\right), \quad \Theta_{j k}(x)=f\left(x ;\left|n_{j}-n_{k}\right|, v_{j} v_{k}\right)+ \\
f\left(x ; n_{j}+n_{k}, v_{j} v_{k}\right)+2 \sum_{i=1}^{\operatorname{Min}\left(n_{j}, n_{k}\right)-1} f\left(x ;\left|n_{j}-n_{k}\right|+2 i, v_{j} v_{k}\right),
\end{array}
$$



a) $\gamma=\pi / 5$
b) $\gamma=4 \pi / 5$
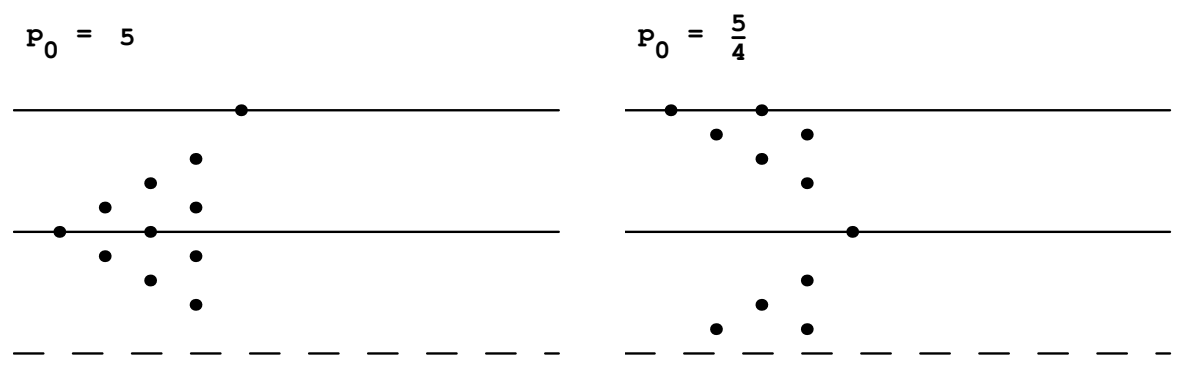

c) $\gamma=3 \pi / 16$

d) $\gamma=13 \pi / 16$

$\mathrm{p}_{0}=\frac{1}{3} \underline{6}$

$p_{0}=\frac{16}{13}$
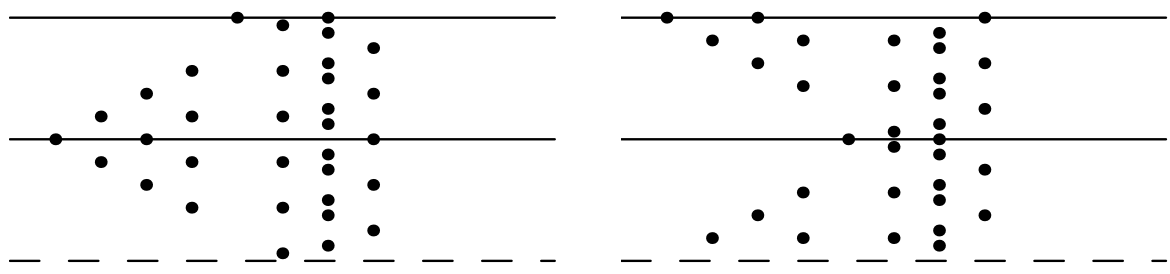

e) $\gamma=13 \pi / 69$

f) $\gamma=56 \pi / 69$

$p_{0}=\frac{69}{13}$

$p_{0}=\frac{69}{5} \frac{9}{6}$
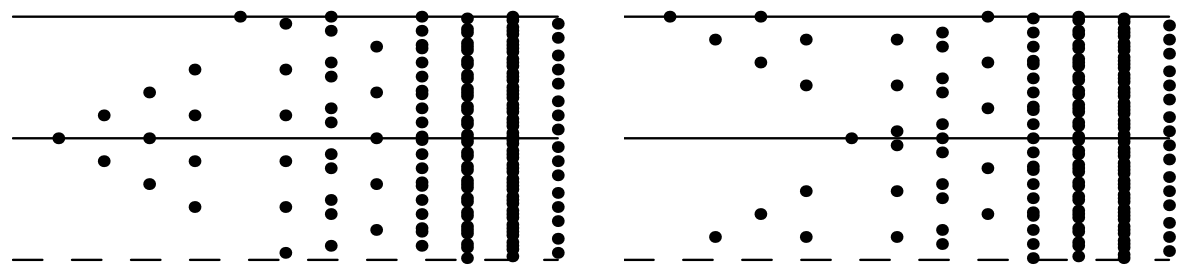

Fig. 1. Strings on the complex plane for given values of $p_{0}=\pi / \gamma$. If we change $\gamma \rightarrow \pi-\gamma$, the strings are almost the same. But imaginary parts of $\gamma x_{j}$ shift by $\pi$. 
and

$$
f(x ; n, v) \equiv\left\{\begin{array}{c}
0 \quad \text { for } n / p_{0}=\text { integer, } \\
2 v \tan ^{-1}\left\{\left(\cot \left(n \pi / 2 p_{0}\right)\right)^{v} \tanh \left(\pi x / 2 p_{0}\right)\right\} \text { otherwise. }
\end{array}\right.
$$

The quantity $I_{\alpha}^{j}$ is an integer (half-odd integer) for $M_{j}$ odd (even), which is located in the region

$$
\left|I_{\alpha}^{j}\right|<\frac{1}{2 \pi}\left|N \theta_{j}(\infty)-\sum_{k=1}^{m_{l}} M_{k} \Theta_{j, k}(\infty)\right| .
$$

The function $f\left(x ; n_{j}, v_{j}\right)$ is a monotonically increasing function for $m_{2 i} \leq j<$ $m_{2 i+1}$ and a monotonically decreasing function for $m_{2 i-1} \leq j<m_{2 i}$.

\subsection{Bethe-ansatz equation for XXZ model with $|\Delta|<1$}

Following Yang and Yang we define particles and holes of strings. We obtain an integral equation for distribution functions $\rho_{j}$ and $\rho_{j}^{h}$ of particles and holes of strings in the thermodynamic limit,

$$
a_{j}(x)=\operatorname{sign}\left(q_{j}\right)\left(\rho_{j}(x)+\rho_{j}^{h}(x)\right)+\sum_{k=1}^{m_{l}} T_{j, k} * \rho_{k}(x) .
$$

Here

$$
a_{j}(x) \equiv(2 \pi)^{-1} \frac{d}{d x} \theta_{j}(x), \quad T_{j, k}(x) \equiv(2 \pi)^{-1} \frac{d}{d x} \Theta_{j, k}(x) .
$$

The symbol $a * b$ denotes the convolution of $a(x)$ and $b(x)$ as follows

$$
a * b(x)=\int_{-\infty}^{\infty} a(x-y) b(y) d y .
$$

The functions $a_{j}(x)$ and their Fourier transforms $\tilde{a}_{j}(\omega)$ are written as

$$
\begin{aligned}
a_{j}(x) & =\frac{1}{2 \pi} \frac{\gamma \sin \gamma q_{j}}{\cosh \gamma x+\cos \gamma q_{j}}, \quad \tilde{a}_{j}(\omega)=\frac{\sinh q_{j} \omega}{\sinh p_{0} \omega}, \\
q_{j} & \equiv(-1)^{i}\left(p_{i}-\left(j-m_{i}\right) p_{i+1}\right), \text { for } m_{i} \leq j<m_{i+1},
\end{aligned}
$$

where the $p_{i}, \quad i=0,1, \ldots, l$ are defined by

$$
p_{0}=\pi / \gamma, \quad p_{1}=1, \quad \nu_{i}=\left[p_{i-1} / p_{i}\right], \quad p_{i}=p_{i-2}-p_{i-1} \nu_{i-1} .
$$

For the series $q_{j}$ we find the following relations

$$
\begin{array}{r}
q_{j}=\frac{1}{2}\left[\left(1-2 \delta_{m_{i}, j}\right) q_{j-1}+q_{j+1}\right], \text { for } m_{i} \leq j \leq m_{i+1}-2, \\
q_{j}=\left(1-2 \delta_{m_{i-1}, j}\right) q_{j-1}+q_{j+1}, \quad \text { for } j=m_{i}-1, \quad i<l \\
q_{0}=p_{0}, \quad n_{m_{l}}+n_{m_{l}-1}=0 .
\end{array}
$$


The Fourier transform of $T_{j, k}(x)$ is given by

$$
\begin{aligned}
& \tilde{T}_{j, k}(\omega)=\tilde{T}_{k, j}(\omega) \\
& =2 \operatorname{sign}\left(q_{j}\right) \operatorname{coth}\left(p_{i+1} \omega\right) \frac{\sinh \left(\left(p_{0}-\left|q_{j}\right|\right) \omega\right) \sinh \left(q_{k} \omega\right)}{\sinh \left(p_{0} \omega\right)} \\
& +\delta_{j, m_{l}-1} \delta_{k, m_{l}}-\delta_{j, k}, \quad \text { for } j \leq k, \quad m_{i}<j \leq m_{i+1} .
\end{aligned}
$$

At $j=1$ we have

$$
\tilde{T}_{1, k}(\omega)=\operatorname{sign}\left(q_{1}\right) 2 \cosh \omega \tilde{a}_{k}(\omega)-\delta_{1, k} .
$$

At $j=m_{l}$ we have

$$
\begin{aligned}
& \tilde{T}_{m_{l}, k}(\omega)=-\tilde{T}_{m_{l}-1, k}(\omega), \\
& \tilde{T}_{m_{l}, m_{l}}(\omega)=-\tilde{T}_{m_{l}-1, m_{l}}(\omega)=\frac{\sinh \left(\left(p_{0}-2 p_{l}\right) \omega\right)}{\sinh \left(p_{0} \omega\right)} .
\end{aligned}
$$

The energy and entropy per site are given by

$$
\begin{aligned}
& e=-\left(\frac{J \Delta}{4}+h\right)+\sum_{j=1}^{m_{l}} \int_{-\infty}^{\infty} g_{j}(x) \rho_{j}(x) d x, \\
& g_{j}(x) \equiv-\frac{2 \pi J \sin \gamma}{\gamma} a_{j}(x)+2 n_{j} h
\end{aligned}
$$

and

$$
s=\int_{-\infty}^{\infty} \rho_{j} \ln \left(1+\frac{\rho_{j}^{h}}{\rho_{j}}\right)+\rho_{j}^{h} \ln \left(1+\frac{\rho_{j}}{\rho_{j}^{h}}\right) d x .
$$

To minimize the free energy density $e-T s$ with respect to $\rho_{j}$, we have

$$
\delta(e-T s)=\sum_{j} \int_{-\infty}^{\infty} g_{j}(x) \delta \rho_{j}(x)-T\left\{\delta \rho_{j} \ln \left(1+\frac{\rho_{j}^{h}}{\rho_{j}}\right)+\delta \rho_{j}^{h} \ln \left(1+\frac{\rho_{j}}{\rho_{j}^{h}}\right)\right\} d x .
$$

The variation of (218) gives

$$
\delta \rho_{j}^{h}=-\delta \rho_{j}-\operatorname{sign}\left(q_{j}\right) \sum T_{j k} * \delta \rho_{k} .
$$

Thus we obtain

$$
\begin{aligned}
& \delta(e-T s)=T \sum_{j} \int_{-\infty}^{\infty} d x \delta \rho_{j}(x) \\
& \left\{\frac{g_{j}(x)}{T}-\ln \left(\frac{\rho_{j}^{h}}{\rho_{j}}\right)+\sum_{k} \operatorname{sign}\left(q_{k}\right) T_{j, k} * \ln \left(1+\frac{\rho_{k}}{\rho_{k}^{h}}\right)\right\} .
\end{aligned}
$$


At the thermodynamic equilibrium one obtains the following non-linear equations determining $\eta_{j}(x) \equiv \rho_{j}^{h}(x) / \rho_{j}(x)$,

$$
\ln \eta_{j}(x)=g_{j}(x) / T+\sum_{k=1}^{m_{l}} \operatorname{sign}\left(q_{k}\right) T_{k, j} * \ln \left(1+\eta_{k}^{-1}(x)\right), \quad j=1, \ldots, m_{l} .
$$

The free energy is given as follows,

$$
\begin{gathered}
f=e-T s=-\left(\frac{J \Delta}{4}+h\right) \\
+\sum_{j=1}^{m_{l}} \int_{-\infty}^{\infty} \rho_{j}(x)\left[g_{j}(x)-T \ln \eta_{j}(x)\right]-T\left[\rho_{j}+\rho_{j}^{h}\right] \ln \left(1+\eta_{j}^{-1}\right) .
\end{gathered}
$$

If we substitute (230) into the first bracket and (218) into the second, the $T_{j, k}$ terms are cancelled and we get

$$
f=-\left(\frac{J \Delta}{4}+h\right)-T \sum_{j=1}^{m_{l}} \operatorname{sign}\left(q_{j}\right) \int_{-\infty}^{\infty} a_{j}(x) \ln \left(1+\eta_{j}^{-1}(x)\right) d x .
$$

If one uses the $j=1$ case of equation (230) and (226), one obtains

$$
\begin{aligned}
& f=-\frac{J \Delta}{4}-\operatorname{sign}\left(q_{1}\right) \frac{2 \pi J \sin \gamma}{\gamma} \int_{-\infty}^{\infty} a_{1}(x) s_{1}(x) d x \\
& -T \int_{-\infty}^{\infty} s_{1}(x) \ln \left(1+\eta_{1}(x)\right) d x
\end{aligned}
$$

From equation (221-223) we get the following relations,

$$
\begin{aligned}
& a_{j}-s_{i} *\left(\left(1-2 \delta_{m_{i-1}, j}\right) a_{j-1}+a_{j+1}\right)=0 \\
& \text { for } m_{i-1} \leq j \leq m_{i}-2, \\
& a_{m_{i}-1}-\left(1-2 \delta_{m_{i-1}, m_{i}-1}\right) s_{i} * a_{m_{i}-2}-d_{i} * a_{m_{i}-1}-s_{i+1} * a_{m_{i}}=0 \\
& \text { for } i<l \\
& a_{m_{l}-1}(x)=-a_{m_{l}}(x)=s_{l} * a_{m_{l}-2},
\end{aligned}
$$

where

$$
\begin{aligned}
& a_{0}(x)=\delta(x), \\
& s_{i}(x) \equiv \int_{-\infty}^{\infty} \frac{d \omega}{4 \pi} \frac{e^{i \omega x}}{\cosh \left(p_{i} \omega\right)}=\frac{1}{4 p_{i}} \operatorname{sech} \frac{\pi x}{2 p_{i}}, \\
& d_{i}(x) \equiv \int_{-\infty}^{\infty} \frac{d \omega}{4 \pi} \frac{e^{i \omega x} \cosh \left(\left(p_{i}-p_{i+1}\right) \omega\right)}{\cosh \left(p_{i} \omega\right) \cosh \left(p_{i+1} \omega\right)} .
\end{aligned}
$$


Using (225) one can show the following relations,

$$
\begin{aligned}
& T_{j, k}-s_{i} *\left(\left(1-2 \delta_{m_{i-1}, j}\right) T_{j-1, k}+T_{j+1, k}\right) \\
& =(-1)^{i+1}\left(\delta_{j-1, k}+\delta_{j+1, k}\right) s_{i}, \\
& \quad \text { for } m_{i-1} \leq j \leq m_{i}-2, \\
& T_{m_{i}-1, k}-\left(1-2 \delta_{m_{i-1}, m_{i}-1}\right) s_{i} * T_{m_{i}-2, k}-d_{i} * T_{m_{i}-1, k} \\
& -s_{i+1} * T_{m_{i}, k}=(-1)^{i+1}\left(\delta_{m_{i}-2, k} s_{i}+\delta_{m_{i}-1, k} d_{i}-\delta_{m_{i}, k} s_{i+1}\right), \\
& \text { for } i=1,2, ., l-1, \\
& T_{m_{l}-1, k}=-T_{m_{l}, k}=s_{l} * T_{m_{l}-2, k}+\operatorname{sign}\left(q_{k}\right) \delta_{m_{l}-2, k} s_{l},
\end{aligned}
$$

with $T_{0, k}=0$. Using (233) and (235) one can rewrite (218) as follows

$$
\begin{aligned}
& \rho_{j}+\rho_{j}^{h}=s_{i} *\left(\rho_{j-1}^{h}+\rho_{j+1}^{h}\right) \text { for } m_{i-1} \leq j \leq m_{i}-2, \\
& \rho_{m_{i}-1}+\rho_{m_{i}-1}^{h}=s_{i} * \rho_{m_{i}-2}^{h}+d_{i} * \rho_{m_{i}-1}^{h}-s_{i+1} * \rho_{m_{i}}^{h}, \\
& \rho_{m_{l}-1}+\rho_{m_{l}-1}^{h}=\rho_{m_{l}}+\rho_{m_{l}}^{h}=s_{l} * \rho_{m_{l}-1}^{h},
\end{aligned}
$$

with $\rho_{0}^{h}=\delta(x)$. Equations (230) are rewritten as

$$
\begin{aligned}
& \ln \left(1+\eta_{0}\right)=-\frac{2 \pi J \sin \gamma}{\gamma T} \delta(x), \\
& \ln \eta_{j}=\left(1-2 \delta_{m_{i-1}, j}\right) s_{i} * \ln \left(1+\eta_{j-1}\right)+s_{i} * \ln \left(1+\eta_{j+1}\right), \\
& \text { for } m_{i-1} \leq j \leq m_{i}-2, j \neq m_{l}-2 \\
& \ln \eta_{m_{i}-1}=\left(1-2 \delta_{m_{i-1}, m_{i}-1}\right) s_{i} * \ln \left(1+\eta_{m_{i}-2}\right) \\
& +d_{i} * \ln \left(1+\eta_{m_{i}-1}\right)+s_{i+1} * \ln \left(1+\eta_{m_{i}}\right), \text { for } i<l \\
& \ln \eta_{m_{l}-2}=\left(1-2 \delta_{m_{l-1}, m_{l}-2}\right) s_{l} * \ln \left(1+\eta_{m_{l}-3}\right) \\
& +s_{l} * \ln \left(\left(1+\eta_{m_{l}-1}\right)\left(1+\eta_{m_{l}}^{-1}\right)\right), \\
& \ln \eta_{m_{l}-1}-y_{l} h / T=y_{l} h / T-\ln \eta_{m_{l}} \\
& =s_{l} * \ln \left(1+\eta_{m_{l}-2}\right) .
\end{aligned}
$$

Then if we write $\ln \kappa(x)=\ln \eta_{m_{l}-1}-y_{l} h / T$ we have integral equations with $m_{l}-1$ unknown functions

$$
\begin{aligned}
& \ln \left(1+\eta_{0}\right)=-\frac{2 \pi J \sin \gamma}{\gamma T} \delta(x), \\
& \ln \eta_{j}=\left(1-2 \delta_{m_{i-1}, j}\right) s_{i} * \ln \left(1+\eta_{j-1}\right)+s_{i} * \ln \left(1+\eta_{j+1}\right), \\
& \text { for } m_{i-1} \leq j \leq m_{i}-2, j \neq m_{l}-2 \\
& \ln \eta_{m_{i}-1}=\left(1-2 \delta_{m_{i-1}, m_{i}-1}\right) s_{i} * \ln \left(1+\eta_{m_{i}-2}\right) \\
& +d_{i} * \ln \left(1+\eta_{m_{i}-1}\right)+s_{i+1} * \ln \left(1+\eta_{m_{i}}\right), \text { for } i<l \\
& \ln \eta_{m_{l}-2}=\left(1-2 \delta_{m_{l-1}, m_{l}-2}\right) s_{l} * \ln \left(1+\eta_{m_{l}-3}\right) \\
& +s_{l} * \ln \left(1+2 \cosh \left(y_{l} h / T\right) \kappa+\kappa^{2}\right) \\
& \ln \kappa(x)=s_{l} * \ln \left(1+\eta_{m_{l}-2}\right) .
\end{aligned}
$$




\section{Some special limits}

\section{$17.1 \quad T \rightarrow \infty$ or $J \rightarrow 0$ limit}

In equations (238), $\ln \left(1+\eta_{0}\right)$ becomes zero and $\eta_{j}(x)$ are all independent of $x$. This yields the following difference equation

$$
\begin{aligned}
& \eta_{j}^{2}=\left(1+\eta_{j-1}\right)^{1-2 \delta_{m_{i-1}, j}}\left(1+\eta_{j+1}\right) \\
& \quad \text { for } m_{i-1} \leq j \leq m_{i}-2, j \neq m_{l}-2 \\
& \eta_{m_{i}-1}^{2}=\left(1+\eta_{m_{i}-2}\right)^{1-2 \delta_{m_{i-1}, m_{i}-1}}\left(1+\eta_{m_{i}-1}\right) \ln \left(1+\eta_{m_{i}}\right), \\
& \quad \text { for } i<l \\
& \eta_{m_{l}-2}^{2}=\left(1+\eta_{m_{l}-3}\right)^{1-2 \delta_{m_{l-1}, m_{l}-2}}\left(1+2 \cosh \left(y_{l} h / T\right) \kappa+\kappa^{2}\right), \\
& \kappa^{2}=\left(1+\eta_{m_{l}-2}\right) .
\end{aligned}
$$

The solution of this set of equations is

$$
\begin{aligned}
& \eta_{j}=\left(\frac{\sinh \left(n_{j}+y_{i-1}\right) h / T}{\sinh \left(y_{i-1} h / T\right)}\right)^{2}-1 \\
& \quad \text { for } m_{i-1}<j \leq m_{i}, \quad j \leq m_{l}-2, \\
& \kappa=\frac{\sinh \left(n_{m_{l}-2}+y_{l}\right) h / T}{\sinh \left(y_{l} h / T\right)} .
\end{aligned}
$$

For $j=1$ we have $\eta_{1}=(2 \cosh h / T)^{2}-1$. Substituting this into (232) we find the free energy

$$
f / T=-\ln (2 \cosh h / T) .
$$

At $h=0$ this gives that entropy per site is $\ln 2$, as it should be.

\section{$17.2 J>0, \quad T \rightarrow 0$ limit}

We define $\epsilon_{j}(x)=T \ln \eta_{j}(x)$ and $\epsilon_{j}^{+}(x)=T \ln \left(1+\eta_{j}(x)\right)$. One can show that $\epsilon_{j}, j \geq 2$ is always positive. The equation (230) gives

$$
\epsilon_{1}(x)=-\frac{2 \pi J \sin \gamma}{\gamma} a(x, 1)+2 h-\int_{-\infty}^{\infty} a(x-y, 2) \epsilon_{1}^{-}(y) d y .
$$

If $\epsilon(x)<0$ at $|x|<B$ and $\epsilon(x)>0$ at $|x|>B$, then one obtains a linear integral equation for $\rho_{1}(x)$,

$$
\rho_{1}(x)+\int_{-B}^{B} a(x-y, 2) \rho_{1}(y) d y=a(x, 1),
$$

where

$$
a(x, n) \equiv \frac{1}{2 \pi} \frac{\gamma \sin n \gamma}{\cosh \gamma x-\cos n \gamma}
$$




\section{Thermodynamics of the XYZ model}

\section{Bethe-ansatz equations for the XYZ model.}

Here we consider the symmetry of the following Hamiltonian

$$
\mathcal{H}=-\sum_{l=1}^{N} J_{x} S_{l}^{x} S_{l+1}^{x}+J_{y} S_{l}^{y} S_{l+1}^{y}+J_{x} S_{l}^{z} S_{l+1}^{z} .
$$

We assume $N$ is even. By the transformation

$$
U_{2} \mathcal{H} U_{2}^{-1}, \quad U_{2}=\prod_{l=\text { even }} 2 S_{l}^{z},
$$

$\mathcal{H}\left(J_{x}, J_{y}, J_{z}\right) \rightarrow \mathcal{H}\left(-J_{x},-J_{y}, J_{z}\right)$. In the same way

$\mathcal{H}\left(J_{x}, J_{y}, J_{z}\right) \rightarrow \mathcal{H}\left(J_{x},-J_{y},-J_{z}\right)$ and $\mathcal{H}\left(J_{x}, J_{y}, J_{z}\right) \rightarrow \mathcal{H}\left(J_{x},-J_{y},-J_{z}\right)$. Namely the energy spectrum of this Hamiltonian is unchanged for reversing signs of two $J_{\alpha}$ 's. It is evident that the spectrum is unchanged for exchanging $J_{\alpha}$ 's. Thus it is sufficient to treat only the case $1 \geq J_{y} / J_{z} \geq\left|J_{x}\right| / J_{z} \geq 0$. Baxter solved the eight-vertex model and also the XYZ model (Baxter (1972a), Baxter (1972b)). The Bethe ansatz equation for this model is

$$
\begin{aligned}
& \left(\frac{H_{l}\left(i \zeta\left(x_{l}+i\right)\right)}{H_{l}\left(i \zeta\left(x_{l}-i\right)\right)}\right)^{N}=-e^{-2 \pi i \nu^{\prime} / p_{0}} \prod_{j=1}^{N / 2} \frac{H_{l}\left(i \zeta\left(x_{l}-x_{j}+2 i\right)\right)}{H_{l}\left(i \zeta\left(x_{l}-x_{j}-2 i\right)\right)}, \\
& \sum_{l}^{N / 2} x_{l}=Q \nu^{\prime}+i p_{0} \nu, \quad Q=K\left(l^{\prime}\right) / \zeta, \quad p_{0}=K(l) / \zeta .
\end{aligned}
$$

Here the modulus $l$ and the parameter $\zeta$ are determined by

$$
l=\sqrt{\frac{J_{z}^{2}-J_{y}^{2}}{J_{z}^{2}-J_{x}^{2}}}, \quad \operatorname{cn}(2 \zeta, l)=-J_{x} / J_{z}
$$

There are $N / 2$ rapidities. $H_{l}(x)$ is the Jacobian elliptic function defined by

$$
H_{l}(x) \equiv 2 \sum_{n=1}^{\infty}(-1)^{n+1} q^{n(n-1)+1 / 4} \sin (2 n-1) \frac{\pi x}{2 K}, \quad q \equiv \exp \left(-\frac{\pi K\left(l^{\prime}\right)}{K(l)}\right) .
$$


This function has the following properties,

$$
H_{l}(x)=-H_{l}(x+2 K(l))=-q e^{i \pi x / K(l)} H_{l}\left(x+2 i K\left(l^{\prime}\right)\right) .
$$

In the Bethe ansatz equation (166), the function $\sinh \frac{\gamma}{2} x$ is merely replaced by the elliptic theta function. The energy is given by

$$
E=-\frac{N J_{z}}{4}\left[1-\frac{\pi \operatorname{sn} 2 \zeta}{\zeta}(\mathbf{a}(0,1)+\mathbf{a}(Q, 1))\right]-\frac{J_{z} \pi \operatorname{sn} 2 \zeta}{\zeta} \sum_{l=1}^{N / 2} \mathbf{a}\left(x_{l}, 1\right)
$$

where

$$
\mathbf{a}(x, l) \equiv \frac{1}{2 \pi i} \frac{d}{d x} \ln \left(\frac{H_{l}(i \zeta(x+i l))}{H_{l}(i \zeta(x-i l))}\right) .
$$

\section{Strings and the thermodynamic Bethe ansatz equation for the XYZ model}

In the limit $l \rightarrow 0$ equation (246) becomes

$$
\left(\frac{\sin \left(i \zeta\left(x_{l}+i\right)\right)}{\sin \left(i \zeta\left(x_{l}-i\right)\right)}\right)^{N}=-e^{-2 \pi i \nu^{\prime} / p_{0}} \prod_{j=1}^{N / 2} \frac{\sin \left(i \zeta\left(x_{l}-x_{j}+2 i\right)\right)}{\sin \left(i \zeta\left(x_{l}-x_{j}-2 i\right)\right)} .
$$

This equation is equivalent to (166), if we assume that the $x_{l}$ 's are finite and $\nu^{\prime}=0$. $\zeta$ becomes $\gamma / 2$ and $K_{l}$ becomes $\pi / 2$. So it is natural to assume the same types of strings can be determined using $p_{0}=K_{l} / \zeta$,

$$
\begin{aligned}
& x_{j}=\alpha+(n+1-2 j) i, \\
& x_{j}=\alpha+(n+1-2 j) i+p_{0} i, \quad Q \geq \alpha>-Q .
\end{aligned}
$$

The solution becomes doubly periodic. So we consider the distribution of solutions at $-Q<\Re x \leq Q$ and $-p_{0}<\Im x \leq p_{0}$. It is expected that the same kind of strings appear in the case of XXZ model at $\pi / \gamma=p_{0}$. We can determine $n_{j}$ 's and $q_{j}$ 's via (209) and (222),

$$
\begin{aligned}
& \left\{e_{j}\left(x_{\alpha}^{j}\right)\right\}^{N}=-\exp \left(-2 \pi i \nu^{\prime} / p_{0}\right) \prod_{k=1}^{m_{l}} \prod_{\beta=1}^{M_{k}} E_{j k}\left(x_{\alpha}^{j}-x_{\beta}^{k}\right), \\
& \nu^{\prime}=\frac{1}{Q} \sum_{j=1}^{m_{l}} \sum_{\alpha=1}^{M_{j}} n_{j} x_{\alpha}^{j},
\end{aligned}
$$


where

$$
\begin{aligned}
& e_{j}(x)=g\left(x ; n_{j}, v_{j}\right), \\
& g(x ; n,+)=\frac{H_{l}(i \zeta(x+i n))}{H_{l}(i \zeta(x-i n))}, \\
& g(x ; n,-)=-\frac{H_{l}\left(K_{l}+i \zeta(x+i n)\right)}{H_{l}\left(K_{l}+i \zeta(x-i n)\right)}, \\
& E_{j k}(x)=\left\{\begin{array}{c}
g\left(x ; 2 n_{j}, v_{j} v_{k}\right) \prod_{l=1}^{n_{j}-1} g^{2}\left(x ; 2 l, v_{j} v_{k}\right) \text { for } n_{j}=n_{k}, \\
g\left(x ;\left(n_{j}+n_{k}\right), v_{j} v_{k}\right) g\left(x ;\left|n_{j}-n_{k}\right|, v_{j} v_{k}\right) \\
\times \prod_{l=1}^{M i n\left(n_{j}, n_{k}\right)-1} g^{2}\left(x ;\left|n_{j}-n_{k}\right|+2 l, v_{j} v_{k}\right) \\
\text { for } n_{j} \neq n_{k},
\end{array}\right.
\end{aligned}
$$

Taking the logarithm of (252) we have

$$
N \theta_{j}\left(x_{\alpha}^{j}\right)=2 \pi I_{\alpha}^{j}-2 \pi \nu^{\prime} / p_{0}+\sum_{k=1}^{m_{l}} \sum_{\beta=1}^{M_{k}} \Theta_{j k}\left(x_{\alpha}^{j}-x_{\beta}^{k}\right), \quad \alpha=1,2, \ldots, M_{j} .
$$

Here

$$
\begin{aligned}
& \theta_{j}(x)=\mathbf{f}\left(x ; n_{j}, v_{j}\right), \quad \Theta_{j k}(x)=\mathbf{f}\left(x ;\left|n_{j}-n_{k}\right|, v_{j} v_{k}\right)+ \\
& \mathbf{f}\left(x ; n_{j}+n_{k}, v_{j} v_{k}\right)+2 \sum_{i=1}^{\operatorname{Min}\left(n_{j}, n_{k}\right)-1} \mathbf{f}\left(x ;\left|n_{j}-n_{k}\right|+2 i, v_{j} v_{k}\right),
\end{aligned}
$$

and $\mathbf{f}(x, n, v)$ is defined by

$$
\mathbf{f}(x, n, v)=f(x, n, v)+\sum_{l=1}^{\infty} f(x-2 l Q, n, v)+f(x+2 l Q, n, v) .
$$

$f(x, n, v)$ was defined in (216). An eigenstate should be identified by the set of quantum numbers $I_{j}^{\alpha}$. From (248) the energy must be

$$
\begin{aligned}
& E=-N J_{z} R-\frac{J_{z} \pi \operatorname{sn} 2 \zeta}{\zeta} \sum_{l=1}^{N / 2} \mathbf{a}\left(x_{l}, 1\right)=-N J_{z} R-\frac{J_{z} \pi \operatorname{sn} 2 \zeta}{\zeta} \sum_{j=1}^{m_{l}} \sum_{\alpha=1}^{M_{j}} \mathbf{a}_{j}\left(x_{\alpha}\right), \\
& R \equiv \frac{1}{4}\left[1-\frac{\pi \operatorname{sn} 2 \zeta}{\zeta}(\mathbf{a}(0,1)+\mathbf{a}(Q, 1))\right] \\
& \mathbf{a}_{j}(x) \equiv \frac{1}{2 Q}\left[\frac{q_{j}}{p_{0}}+2 \sum_{l=1}^{\infty} \frac{\sinh \left(q_{j} \pi l / Q\right)}{\sinh \left(p_{0} \pi l / Q\right)} \cos (\pi j x / Q)\right]
\end{aligned}
$$

where $q_{j}$ was defined in (222) The number of zeros must be $N / 2$, so

$$
N / 2=\sum_{j=1}^{m_{l}} n_{j} M_{j} .
$$


Thus the energy per site is given by

$$
e=-J_{z} R-\frac{J_{z} \pi \mathrm{sn} 2 \zeta}{\zeta} \sum_{j=1}^{m_{l}} \int_{-Q}^{Q} \mathbf{a}_{j}(x) \rho_{j}(x) d x .
$$

The entropy per site is

$$
s=\sum_{j=1}^{m_{l}} \int_{-Q}^{Q} \rho_{j} \ln \left(1+\frac{\rho_{j}^{h}}{\rho_{j}}\right)+\rho_{j}^{h} \ln \left(1+\frac{\rho_{j}}{\rho_{j}^{h}}\right) d x .
$$

From (257) we have the relation between $\rho_{j}(x)$ and $\rho_{j}^{h}(x)$,

$$
\mathbf{a}_{j}(x)=\operatorname{sign}\left(q_{j}\right)\left(\rho_{j}(x)+\rho_{j}^{h}(x)\right)+\sum_{k=1}^{m_{l}} \mathbf{T}_{j, k} * \rho_{k}(x) .
$$

Moreover, from (259),

$$
\frac{1}{2}=m \equiv \sum_{j=1}^{m_{l}} n_{j} \int_{-Q}^{Q} \rho_{j}(x) d x .
$$

Next we need a Lagrange multiplier to guarantee the condition (263). One should minimize $e-T s+2 h m$ under conditions (262), and after that the multiplier $h$ should be chosen so that (263) is satisfied. Just in the same way as before we get the integral equations for $\eta_{j}(x)=\rho_{j}^{h}(x) / \rho_{j}(x)$,

$$
\ln \eta_{j}(x)=\mathbf{g}_{j}(x) / T+\sum_{k=1}^{m_{l}} \operatorname{sign}\left(q_{k}\right) \mathbf{T}_{k, j} * \ln \left(1+\eta_{k}^{-1}(x)\right), \quad j=1, \ldots, m_{l} .
$$

Here $*, \mathbf{g}_{j}(x)$ and $\mathbf{T}_{j, k}(x)$ are

$$
\begin{gathered}
f * g(x)=\int_{-Q}^{Q} f(x-y) g(y) d y, \\
\mathbf{g}_{j}(x) \equiv-\frac{J_{z} \pi \mathrm{sn} 2 \zeta}{\zeta} \mathbf{a}_{j}(x)+2 n_{j} h, \\
\mathbf{T}_{j, k}(x)=\frac{1}{2 Q} \sum_{l=-\infty}^{\infty} e^{i \pi n x / Q} \tilde{T}_{j, k}\left(\frac{\pi l}{Q}\right)=\sum_{l=-\infty}^{\infty} T_{j, k}(x-2 l Q) .
\end{gathered}
$$

The quantity $g \equiv e-T s+2 h m$ is given as follows

$$
\begin{aligned}
& g\left(J_{z}, T, h\right)=-J_{z} R \\
& +\sum_{j=1}^{m_{l}} \int_{-Q}^{Q} \rho_{j}(x)\left[\mathbf{g}_{j}(x)-T \ln \eta_{j}(x)\right]-T\left[\rho_{j}+\rho_{j}^{h}\right] \ln \left(1+\eta_{j}^{-1}\right) d x \\
& =-J_{z} R-T \sum_{j=1}^{m_{l}} \operatorname{sign}\left(q_{j}\right) \int_{-Q}^{Q} \mathbf{a}_{j}(x) \ln \left(1+\eta_{j}^{-1}(x)\right) d x .
\end{aligned}
$$


Corresponding to (232) this is

$$
\begin{aligned}
& g=-J_{z} R+h-\operatorname{sign}\left(q_{1}\right) \frac{\pi J_{z} \operatorname{sn} 2 \zeta}{\zeta} \int_{-Q}^{Q} \mathbf{a}_{1}(x) \mathbf{s}_{1}(x) d x \\
& -T \int_{-Q}^{Q} \mathbf{s}_{1}(x) \ln \left(1+\eta_{1}(x)\right) d x .
\end{aligned}
$$

Then $m$ should be determinded by

$$
m=\frac{1}{2} \frac{\partial g}{\partial h}=\frac{1}{2}-\frac{1}{2} \int_{-Q}^{Q} \mathbf{s}_{1}(x)\left(1+\eta_{1}(x)\right)^{-1} \frac{\partial \eta_{1}(x)}{\partial h} d x .
$$

The equation (264) is also equivalent to the following block tridiagonal equations,

$$
\begin{aligned}
& \ln \left(1+\eta_{0}\right)=-\frac{\pi J_{z} \operatorname{sn} 2 \zeta}{\zeta T} \delta(x), \\
& \ln \eta_{j}=\left(1-2 \delta_{m_{i-1}, j}\right) \mathbf{s}_{i} * \ln \left(1+\eta_{j-1}\right)+\mathbf{s}_{i} * \ln \left(1+\eta_{j+1}\right) \\
& \text { for } m_{i-1} \leq j \leq m_{i}-2, j \neq m_{l}-2 \\
& \ln \eta_{m_{i}-1}=\left(1-2 \delta_{m_{i-1}, m_{i}-1}\right) \mathbf{s}_{i} * \ln \left(1+\eta_{m_{i}-2}\right) \\
& +\mathbf{d}_{i} * \ln \left(1+\eta_{m_{i}-1}\right)+\mathbf{s}_{i+1} * \ln \left(1+\eta_{m_{i}}\right) \text { for } i<l \\
& \ln \eta_{m_{l}-2}=\left(1-2 \delta_{m_{l-1}, m_{l}-2}\right) \mathbf{s}_{l} * \ln \left(1+\eta_{m_{l}-3}\right) \\
& +\mathbf{s}_{l} * \ln \left(1+2 \cosh \left(y_{l} h / T\right) \kappa+\kappa^{2}\right) \\
& \ln \kappa=\mathbf{s}_{l} * \ln \left(1+\eta_{m_{l}-2}\right)
\end{aligned}
$$

In this equation parameter $h$ appears only in $\cosh \left(y_{l} h / T\right)$ term. So $\eta_{1}(x, h)$ is even function of $h$ and $\left.\frac{\partial \eta_{1}(x)}{\partial h}\right|_{h=0}=0$. Using (267) we find $m=1 / 2$ and the condition (263) is satisfied at $h=0$. Thus equation (268) becomes

$$
\begin{aligned}
& \ln \left(1+\eta_{0}\right)=-\frac{\pi J_{z} \operatorname{sn} 2 \zeta}{\zeta T} \delta(x), \\
& \ln \eta_{j}=\left(1-2 \delta_{m_{i-1}, j}\right) \mathbf{s}_{i} * \ln \left(1+\eta_{j-1}\right)+\mathbf{s}_{i} * \ln \left(1+\eta_{j+1}\right) \\
& \text { for } m_{i-1} \leq j \leq m_{i}-2, j \neq m_{l}-2 \\
& \ln \eta_{m_{i}-1}=\left(1-2 \delta_{m_{i-1}, m_{i}-1}\right) \mathbf{s}_{i} * \ln \left(1+\eta_{m_{i}-2}\right) \\
& +\mathbf{d}_{i} * \ln \left(1+\eta_{m_{i}-1}\right)+\mathbf{s}_{i+1} * \ln \left(1+\eta_{m_{i}}\right) \text { for } i<l \\
& \ln \eta_{m_{l}-2}=\left(1-2 \delta_{m_{l-1}, m_{l}-2}\right) \mathbf{s}_{l} * \ln \left(1+\eta_{m_{l}-3}\right) \\
& +2 \mathbf{s}_{l} * \ln (1+\kappa) \\
& \ln \kappa=\mathbf{s}_{l} * \ln \left(1+\eta_{m_{l}-2}\right) .
\end{aligned}
$$

Corresponding to (232) the free energy is

$$
f=-J_{z} R-\operatorname{sign}\left(q_{1}\right) \frac{\pi J_{z} \operatorname{sn} 2 \zeta}{\zeta} \int_{-Q}^{Q} \mathbf{a}_{1}(x) \mathbf{s}_{1}(x) d x
$$




$$
-T \int_{-Q}^{Q} \mathbf{s}_{1}(x) \ln \left(1+\eta_{1}(x)\right) d x .
$$

We can calculate free energy of XYZ model in zero external field. 


\section{Numerical calculation and recent developments}

In this lecture we restricted ourselves to the unnested Bethe ansatz. Fermions with $\delta$-function interactions and the Hubbard model belong to the nested Bethe ansatz which has several kinds of rapidities. For details see (Takahashi (1971b), Lai (1971), Takahashi (1972)). But the method of derivation is essentially the same as the unnested cases.

The equations introduced in this lecture note have been solved numerically. One can calculate the specific heat, magnetic susceptibility, magnetization curve for the XXZ model, and the specific heat for the XYZ model at $p_{0}=2,3$ (Takahashi (1974a)). If we increase $p_{0}$ the problem approaches to the $\Delta=-1$ case. For the analysis of thermodynamic quantities not at low temperature, we can use the high-temperature expansion method or exact diagonalization. For the investigation of low-temperature thermodynamics of solvable models the Bethe ansatz method is the only way. For the spin $1 / 2$ ferromagnetic XXX chain, the susceptibility diverges as $T^{-\gamma}$ and the specific heat behaves as $T^{-\alpha}$. The estimations of exponent $\gamma$ had been done by many authors. Baker et. al. estimated $\gamma=1.66$ using high temperature series expansions (Baker et al (1964)). Lyklema obtained $\gamma=1.75$ using quantum Monte Carlo calculations (Lyklema (1983)). By the numerical calculation of thermodynamic Bethe ansatz equations it was established that $\gamma=2$ and $\alpha=1 / 2$ (Takahashi and Yamada (1985), Yamada and Takahashi (1986), Schlottmann (1985)). This investigation continued to the spinwave theory for low-dimensional magnets (Takahashi (1986), Takahashi (1987), Takahashi (1989)).

In many cases of one-dimensional quantum systems, one can define the quantum transfer matrix. The largest eigenvalue of this matrix gives the free energy. The ratio of the largest and the second largest eigenvalues gives the correlation length. This method was developed mainly by Japanese theorists within the last ten years. The XYZ model and the Hubbard model were investigated by this method, for other solvable models this method is expected to be applicable. Logarithmic anomalies in the low-temperature susceptibility of the XXX antiferromagnet were found by the numerical calculation of Bethe ansatz equations for the largest eigenvalue of the quantum transfer matrix (Eggert, Affleck and Takahashi (1994)). 


\section{References}

Baker Jr., G.A., Rushbrooke G.S. and Gilbert, H.E. (1964) Phys. Rev. 135 A 1272.

Baxter, R.(1972a). Ann. Phys.(N.Y.) 70, 193.

Baxter, R.(1972b). Ann. Phys.(N.Y.) 70, 323.

Bethe, H.A.(1931). Z. Physik 71, 205.

Bonner J.C. and Fisher,M.E. (1964). Phys. Rev.135, A640

des Cloizeaux, J. and Pearson, J.(1962). Phys. Rev. 128, 2131.

Eggert, S. Affleck, I. and Takahashi, M. (1994) Phys. Rev. Lett. 73332.

Fowler, M. and Zotos, X.(1981) Phys. Rev. B 24,2634.

Gaudin, M.(1971). Phys. Rev. Lett. 26, 1301.

Griffiths, R.B.(1964). Phys. Rev. 133A, 768.

Hida, K. (1981) Phys. Lett. 84A,338.

Hulthen, L.(1938). Arkiv Math. Astron. Fys. 26A, No11.

Katsura, S. (1962). Phys. Rev. 127, 1508.

Katsura, S. (1965). Ann. of Phys. 31, 325.

Lai, C.K.(1971). Phys. Rev. Lett. 26, 1472.

Lieb, E.H.(1963). Phys. Rev. 130, 1616.

Lieb, E.H. and Liniger, W.(1963). Phys. Rev. 130, 1605.

Lieb, E.H., Schultz, T. and Mattis, D. (1961). Ann. Phys. (N.Y.) 16, 417.

Lyklema, J.W. (1983) Phys. Rev. 27, 3108.

Orbach, R.(1958). Phys. Rev. 112, 309.

Schlottmann, P.(1985). Phys. Rev. Lett. 54, 2131.

Schlottmann, P.(1986). Phys. Rev. B 334880 .

Takahashi, M.(1971a). Prog. Theor. Phys. 46, 401.

Takahashi, M.(1971b). Prog. Theor. Phys. 46, 1388.

Takahashi, M.(1972). Prog. Theor. Phys. 47, 69.

Takahashi, M.(1973). Prog. Theor. Phys. 50, 1519.

Takahashi, M.(1974a). Prog. Theor. Phys. 51, 1348.

Takahashi, M.(1974b). Prog. Theor. Phys. 52, 103.

Takahashi, M.(1977). J. of Phys. C 10, 1289.

Takahashi, M.(1986). Prog. Theor. Phys. Suppl. 87, 233.

Takahashi, M.(1987). Phys. Rev. Lett.,58 168,

Takahashi, M.(1989) Phys. Rev. B 402494.

Takahashi, M. and Suzuki, M.(1972). Prog. Theor. Phys. 46, 2187.

Takahashi, M. and Yamada, M.(1985). J. Phys. Soc. Jpn. 54, 2808.

Walker, L.R. (1959). Phys. Rev. 1161089.

Yamada, M and Takahashi, M. (1986) J. Phys. Soc. Jpn., 55, 2024.

Yang, C.N.(1967). Phys. Rev. Lett. 19, 1312.

Yang, C.N. and Yang, C.P. (1966). Phys. Rev. 147 303,150 321,327,151 258.

Yang, C.N. and Yang, C.P. (1969). J. Math. Phys. 10, 1115.

Yang, C.P. (1970). Phys. Rev. A 2, 154. 